\title{
VACUUM STATES AND GLOBAL STABILITY OF RAREFACTION WAVES FOR COMPRESSIBLE FLOW*
}

\author{
GUI-QIANG CHEN ${ }^{\dagger}$
}

\begin{abstract}
The global stability of rarefaction waves in a broad class of entropy solutions in $L^{\infty}$ containing vacuum states is proved for the compressible Euler equations for both one-dimensional isentropic and non-isentropic fluids. Rarefaction waves are the unique case that produces the vacuum states late time in the Riemann solutions when the Riemann initial data are away from the vacuum. Such rarefaction waves are also shown to be global attractors of entropy solutions in $L^{\infty}$ with the vacuum whose initial data are an $L^{\infty} \cap L^{1}$ perturbation of those of the rarefaction waves. Since the instability of solutions containing the vacuum states for the compressible Navier-Stokes equations, some techniques are presented to estimate a lower bound of the density for multidimensional viscous non-isentropic fluids with spherical symmetry between a solid core and a free boundary connected to a surrounding vacuum state. Our analysis works for the solutions with arbitrarily large oscillation. In particular, no assumption of small oscillation and $B V$ regularity of entropy solutions is made for the compressible Euler equations.
\end{abstract}

1. Introduction. We are concerned with the global stability of entropy solutions in $L^{\infty}$ containing vacuum states for the compressible Euler equations. Vacuum (i.e. cavitation) states are important physical states in fluid mechanics and often yield certain singularities in the physical systems, which cause essential analytical difficulties (cf. [4, 8, 11, 16, 18, 20, 26, 27, 28, 29, 31, 34, 35, 36, 43]).

In this paper we focus on the stability of rarefaction waves in a broad class of entropy solutions in $L^{\infty}$ containing the vacuum states for the compressible Euler equations for both one-dimensional isentropic and non-isentropic fluids. One of our main motivations for such a study is that rarefaction waves are the unique case that produces the vacuum late time in the Riemann solutions, when the Riemann initial data are away from the vacuum (cf. Courant-Friedrichs [13], Chang-Hsiao [3]). Another motivation is the instability of solutions containing the vacuum states for the compressible Navier-Stokes equations (see Hoff-Serre [20]; also Xin [43]), so that it is important to understand how well the behavior of entropy solutions with the vacuum is for the compressible Euler equations to explore essential differences between the Euler equations and the Navier-Stokes equations.

In Sections 2.1 and 3, we first study the vacuum states and stability of rarefaction waves in a broad class of entropy solutions in $L^{\infty}$ for the system of compressible Euler equations for isentropic fluids with a general pressure law. The existence and compactness of such entropy solutions with the vacuum have been successfully established for the system with a general pressure law in Chen-LeFloch [11]. Also see DiPerna [18], Ding-Chen-Luo [16], Chen [4], Lions-Perthame-Tadmor [28], and LionsPerthame-Souganidis [29] for polytropic $\gamma$-law fluids. The next natural questions are whether rarefaction waves with the vacuum are stable under initial $L^{\infty} \cap L^{1}$ perturbation, which yields the entropy solutions in $L^{\infty}$ in this class, and whether the rarefaction waves are attractors of the entropy solutions, that is, whether the entropy solutions asymptotically tend to the corresponding rarefaction waves. In Section 3, we establish the stability theorem for the rarefaction waves in the broad class of entropy solutions in $L^{\infty}$ with the vacuum satisfying only one Lax entropy inequality corresponding to the mechanical energy, a special entropy. As a corollary, we conclude

\footnotetext{
*Received January 24, 2000.

†Department of Mathematics, Northwestern University, Lunt Hall, 2033 Sheridan Road, Evanston, IL 60208-2730, USA (gqchen@math.northwestern.edu).
} 
that such rarefaction waves are global attractors of entropy solutions in $L^{\infty}$ with the vacuum whose initial data are the $L^{\infty} \cap L^{1}$ perturbation of those of the rarefaction waves, in which arbitrarily large oscillation is allowed.

Our approach is based on the normal traces and Gauss-Green formula for divergence-measure vector fields in $L^{\infty}$ (see [5]) and the techniques developed in Chen-Frid $[6,7]$ for the stability of Riemann solutions, in combination with DiPerna's method [17] for the uniqueness of Riemann solutions in the class of entropy solutions in $B V$ for $2 \times 2$ strictly hyperbolic systems of conservation laws. One of the new difficulties here is that the strict hyperbolicity of the system fails near the vacuum, which yields the singularity of the derivatives of entropy functions, especially the mechanical energy, and the integrability or boundedness of certain quantities related to the mechanical energy must be carefully analyzed near the vacuum, which is naturally ensured in the strictly hyperbolic case. Another difficulty is that the entropy solutions under consideration are only in $L^{\infty}$.

In Sections 2.2 and 4, we extend our analysis in Sections 2.1 and 3 to the system of compressible Euler equations for non-isentropic fluids, which is more complicated. The approach for the isentropic Euler equations does not directly apply here since the essential dependence of solutions on the physical entropy. The approach for the system away from the vacuum as in [6] also fails if one chooses the typical physical entropy $S^{*}$ as an independent theromdynamical variable. This difficulty is overcome by choosing an appropriate function of the typical physical entropy $S^{*}$ as a new variable, in combination with the techniques in [6]. We prove that the rarefaction waves with the vacuum are also stable in the class of entropy solutions in $L^{\infty}$ with large oscillation for the system of compressible non-isentropic Euler equations, whose initial data are the $L^{\infty} \cap L^{1}$ perturbation of the Riemann data, and the rarefaction waves are attractors of the entropy solutions in this class, that is, the entropy solutions asymptotically tend to the rarefaction waves.

The instability of solutions containing the vacuum states for the compressible Navier-Stokes equations motivates us to examine further a positive lower bound of the density in the region under consideration. In Section 5, we present some techniques to estimate the lower bound of the density for the Navier-Stokes equations for multidimensional non-isentropic fluids with spherical symmetry between a sold core and a free boundary connected to a surrounding vacuum state. The free boundary connects the compressible non-isentropic fluids to the vacuum state with free stress and zero heat flux. The fluids are initially assumed to fill with a finite volume, zero density at the free boundary, and bounded positive density and temperature between the solid core and the initial position of the free boundary. As an illustration, we describe these techniques by showing that any smooth $C^{2}$ solution does not develop the vacuum between the solid core and the free boundary, and the free boundary expands finitely as time evolves. These techniques have been successfully applied to establishing the existence of global solutions and the finiteness of propagation speed of the free boundary for such a problem in Chen-Krakta [9].

2. Vacuum States, Rarefaction Waves, and Divergence-Measure Fields. In this section we first consider the systems of compressible Euler equations for isentropic and non-isentropic fluids, respectively, which can be written into the conservation form:

$$
\partial_{t} U+\partial_{x} F(U)=0
$$


with initial data

$$
\left.U\right|_{t=0}=U_{0}(x)
$$

We focus our discussion on the vacuum states and rarefaction waves in the entropy solutions of (2.1) to the Riemann problem:

$$
\left.U\right|_{t=0}=R_{0}(x) \equiv \begin{cases}U_{-}, & x<0 \\ U_{+}, & x>0\end{cases}
$$

and to the Cauchy problem that is an initial $L^{\infty} \cap L^{1}(\mathbb{R})$ perturbation of $(2.3)$ :

$$
U_{0}(x) \equiv R_{0}(x)+P_{0}(x), \quad P_{0}(x) \in L^{\infty} \cap L^{1}(\mathbb{R}) .
$$
isfies

An $L^{\infty}$ function $U(x, t)$ is called an entropy solution of (2.1)-(2.2) if $U(x, t)$ sat-

(1). Equation (2.1) and Cauchy data (2.2) in the sense of distributions;

(2). At least one Lax entropy inequality:

$$
\partial_{t} \eta(U)+\partial_{x} q(U) \leq 0 \text { in the sense of distributions, }
$$

corresponding to a convex physical entropy pair $(\eta, q)$ which satisfies

$$
\nabla^{2} \eta(U) \geq 0, \quad \nabla q(U)=\nabla \eta(U) \nabla F(U) .
$$

See Definitions 3.1 and 4.1 .

We start with the isentropic Euler equations.

2.1. Isentropic Euler Equations. The system of compressible Euler equations for isentropic fluids reads:

$$
\left\{\begin{array}{l}
\partial_{t} \rho+\partial_{x} m=0 \\
\partial_{t} m+\partial_{x}\left(\frac{m^{2}}{\rho}+p(\rho)\right)=0
\end{array}\right.
$$

where $\rho$ and $m$ are the density and the mass, respectively, and are in the physical region $\left\{(\rho, m)|\rho \geq 0| m \mid, \leq C_{0} \rho\right\}$ for some $C_{0}>0$. The pressure function $p(\rho)$ is a smooth function in $\rho>0$ (nonvacuum states) satisfying

$$
p(\rho)>0, \quad p^{\prime}(\rho)>0, \quad p^{\prime \prime}(\rho)>0, \quad \text { when } \rho>0,
$$

and

$$
p(0)=0, \quad p^{\prime}(0)=0, \quad \lim _{\rho \rightarrow 0} \frac{\rho p^{(k+1)}(\rho)}{p^{(k)}(\rho)}=c_{k}>0, k=0,1 .
$$

For $\rho>0, v=m / \rho$ is the velocity.

For polytropic $\gamma$-law fluids, the pressure function $p(\rho)=k \rho^{\gamma}, \gamma>1, k>0$, clearly satisfies (2.7)-(2.8).

The eigenvalues of system (2.6) are

$$
\lambda_{j}=m / \rho+(-1)^{j} \sqrt{p^{\prime}(\rho)}, \quad j=1,2,
$$


and the corresponding right-eigenvectors are

$$
r_{j}=\alpha_{j}(\rho)\left(1, \lambda_{j}\right)^{\top}, \quad \alpha_{j}(\rho)=(-1)^{j} \frac{2 \rho \sqrt{p^{\prime}(\rho)}}{\rho p^{\prime \prime}(\rho)+2 p^{\prime}(\rho)},
$$

so that

$$
\nabla \lambda_{j} \cdot r_{j}=1 \text { (genuinely nonlinear), } j=1,2 \text {. }
$$

The Riemann invariants are

$$
w_{j}=\frac{m}{\rho}+(-1)^{j+1} \int_{0}^{\rho} \frac{\sqrt{p^{\prime}(s)}}{s} d s, \quad j=1,2 .
$$

From (2.8)-(2.9), we have

$$
\lambda_{2}-\lambda_{1}=2 \sqrt{p^{\prime}(\rho)} \rightarrow 0, \quad \rho \rightarrow 0 .
$$

Therefore, system (2.6) is strictly hyperbolic in the nonvacuum states $\{(\rho, v) \mid \rho>$ $\left.0,|v| \leq C_{0}\right\}$, and the strict hyperbolicity fails near the vacuum states $\{(\rho, m / \rho) \mid \rho=$ $\left.0,|m / \rho| \leq C_{0}\right\}$.

Now we consider the Riemann problem (2.6) and (2.3) for $U=(\rho, m)$ with constants $\rho_{ \pm} \geq 0$ and $m_{ \pm}$satisfying $\left|m_{ \pm} / \rho_{ \pm}\right| \leq C_{0}<\infty$.

Given a state $U_{0}=\left(\rho_{0}, m_{0}\right)$, we consider possible states $U=(\rho, m)$ that can be connected to state $U_{0}$ on the right by a centered rarefaction wave. Consider the selfsimilar solutions $(\rho, m)(\xi), \xi=x / t$, of the Riemann problem (2.6) and (2.3). Then we have

$$
\frac{d m}{d \xi}-\xi \frac{d \rho}{d \xi}=0, \quad \xi=\lambda_{j}(\rho, m)(\xi), \quad j=1,2
$$

Hence, from (2.10),

$$
\frac{\partial U}{\partial x}=\frac{1}{t} \frac{d U}{d \xi}=\frac{1}{t} r_{j}\left(U\left(\frac{x}{t}\right)\right), \quad j=1,2 .
$$

Therefore, there are two families of centered rarefaction waves corresponding to the $j$ th characteristic families, $j=1,2$, respectively.

For the Riemann problem (2.6) and (2.3) satisfying (2.7)-(2.8) and $w_{j}\left(U_{+}\right) \geq$ $w_{j}\left(U_{-}\right), j=1,2$, there exists a unique piecewise smooth entropy solution $R(x / t)$ containing the vacuum states on the upper half-plane $t>0$ and satisfying

$$
\left\{\begin{array}{l}
w_{1}(R(x / t)) \leq w_{1}\left(U_{+}\right), \quad w_{2}(R(x / t)) \geq w_{2}\left(U_{-}\right), \\
w_{1}(R(x / t))-w_{2}(R(x / t)) \geq 0
\end{array}\right.
$$

These Riemann solutions can be constructed as follows.

If $\rho_{-}>0$ and $\rho_{+}=0$, then there exists a unique $v_{c}$ such that

$$
R(x / t)=\left\{\begin{array}{l}
U_{-}, \quad x / t<\lambda_{1}\left(U_{-}\right) \\
R_{1}(x / t), \quad \lambda_{1}\left(U_{-}\right) \leq x / t \leq v_{c} \\
\text { vacuum, } \quad x / t>v_{c}
\end{array}\right.
$$


where $R_{1}(\xi)$ is the solution of the boundary value problem

$$
R_{1}^{\prime}(\xi)=r_{1}\left(R_{1}(\xi)\right), \quad \xi>\lambda_{1}\left(U_{-}\right) ; \quad R_{1}\left(\lambda_{1}\left(U_{-}\right)\right)=U_{-} .
$$

If $\rho_{-}=0$ and $\rho_{+}>0$, then there exists a unique $\tilde{v}_{c}$ such that

$$
R(x / t)=\left\{\begin{array}{l}
\text { vacuum, } \quad x / t<\tilde{v}_{c}, \\
R_{2}(x / t), \quad \tilde{v}_{c} \leq x / t \leq \lambda_{2}\left(U_{+}\right), \\
U_{+}, \quad x / t>\lambda_{2}\left(U_{+}\right),
\end{array}\right.
$$

where $R_{2}(\xi)$ is the solution of the boundary value problem

$$
R_{2}^{\prime}(\xi)=r_{2}\left(R_{2}(\xi)\right), \quad \xi<\lambda_{2}\left(U_{+}\right) ; \quad R_{2}\left(\lambda_{2}\left(U_{+}\right)\right)=U_{+} .
$$

If $\rho_{ \pm}>0$, there are two cases:

(a). There exist unique $v_{c_{1}}, v_{c_{2}}, v_{c_{1}}<v_{c_{2}}$, such that the Riemann solution has the form:

$$
R(x / t) \equiv \begin{cases}U_{-}, & x / t<\lambda_{1}\left(U_{-}\right), \\ R_{1}(x / t), & \lambda_{1}\left(U_{-}\right) \leq x / t \leq v_{c_{1}}, \\ \operatorname{vacuum}, & v_{c_{1}}<x / t<v_{c_{2}}, \\ R_{2}(x / t), & v_{c_{2}} \leq x / t \leq \lambda_{2}\left(U_{+}\right), \\ U_{+}, & x / t>\lambda_{2}\left(U_{+}\right),\end{cases}
$$

where $R_{1}(\xi)$ and $R_{2}(\xi)$ are the solutions of the boundary value problems $(2.15)$ and (2.17), respectively.

(b). There exists a unique $U_{c}=\left(\rho_{c}, m_{c}\right), \rho_{c}>0$, such that the Riemann solution has the form:

$$
R(x / t) \equiv \begin{cases}U_{-}, & x / t<\lambda_{1}\left(U_{-}\right), \\ R_{1}(x / t), & \lambda_{1}\left(U_{-}\right) \leq x / t \leq \lambda_{1}\left(U_{c}\right), \\ U_{c}, & \lambda_{1}\left(U_{c}\right)<x / t<\lambda_{2}\left(U_{c}\right), \\ R_{2}(x / t), & \lambda_{2}\left(U_{c}\right) \leq x / t \leq \lambda_{2}\left(U_{+}\right), \\ U_{+}, & x / t>\lambda_{2}\left(U_{+}\right),\end{cases}
$$

where $R_{1}(\xi)$ and $R_{2}(\xi)$ are the solutions of the boundary value problems $(2.15)$ and $(2.17)$, respectively.

For case (a), although the Riemann data are nonvacuum states at $t=0$, the vacuum states occur in the Riemann solutions instantaneously as time evolves. Therefore, the vacuum states are generic in invicid compressible fluid flow.

2.2. Non-Isentropic Euler Equations. The system of compressible Euler equations for non-isentropic fluids reads:

$$
\left\{\begin{array}{l}
\partial_{t} \rho+\partial_{x} m=0 \\
\partial_{t} m+\partial_{x}\left(p+\frac{m^{2}}{\rho}\right)=0 \\
\partial_{t} E+\partial_{x}\left(\frac{m}{\rho}(E+p)\right)=0
\end{array}\right.
$$

where $E=\frac{1}{2} \frac{m^{2}}{\rho}+\rho e$, and $\rho, m, p, e$ are the density, the mass, the pressure, and the internal energy of the fluids, respectively, and are in the physical region $\{(\rho, m, p, e) \mid \rho \geq$ 
$\left.0,|m| \leq C_{0} \rho, p \geq 0, e \geq 0\right\}$ for some $C_{0}>0$. The other important physical variables are the temperature $\theta$ and the entropy $S^{*}$. To close system (2.20), one needs the basic thermodynamical principle:

$$
d e=\theta d S^{*}+\frac{p}{\rho^{2}} d \rho .
$$

We then choose $\left(\rho, S^{*}\right)$ as the basic variables to obtain the constitutive equations of state $p=p\left(\rho, S^{*}\right), \theta=\theta\left(\rho, S^{*}\right)$, and $e=e\left(\rho, S^{*}\right)$ satisfying

$$
e_{\rho}\left(\rho, S^{*}\right)=p\left(\rho, S^{*}\right) / \rho^{2}, e_{S^{*}}\left(\rho, S^{*}\right)=\theta\left(\rho, S^{*}\right), \theta_{\rho}\left(\rho, S^{*}\right)=p_{S^{*}}\left(\rho, S^{*}\right) / \rho^{2},
$$

from relation (2.21).

For concreteness, in this section and Section 4, we focus on polytropic ideal fluids:

$$
p\left(\rho, S^{*}\right)=\kappa \rho^{\gamma} e^{S^{*} / c_{v}}, \quad e=c_{v} \theta, \quad p=R \rho e,
$$

where $R$ and $c_{v}$ are positive constants, and $\gamma=1+R / c_{v}$ is the adiabatic exponent in the natural physical interval $\gamma \in(1,2]$.

The discontinuous solutions of (2.20) must satisfy the Clausius inequality:

$$
\partial_{t}(\rho \alpha(S))+\partial_{x}(m \alpha(S)) \geq 0, \quad \alpha^{\prime}(S) \geq 0,
$$

in the sense of distributions to single out the physical solutions.

The eigenvalues of system (2.20) are

$$
\lambda_{j}=m / \rho+(-1)^{\frac{j+1}{2}} c, j=1,3 ; \quad \lambda_{2}=m / \rho,
$$

and the corresponding right-eigenvectors are

$$
r_{j}=\alpha_{j}\left(1, \lambda_{j}, \frac{m}{\rho} \lambda_{j}-\frac{1}{2} \frac{m^{2}}{\rho^{2}}+\frac{p+\rho e}{\rho}\right)^{\top}, \quad \alpha_{j}=\frac{(-1)^{\frac{j+1}{2}} 2 \rho c}{\rho p_{\rho \rho}+2 p_{\rho}}, j=1,3,
$$

and $r_{2}=\left(1, m / \rho, m^{2} /\left(2 \rho^{2}\right)\right)$ so that $\nabla \lambda_{2} \cdot r_{2} \equiv 0$ (linearly degenerate), and

$$
\nabla \lambda_{j} \cdot r_{j}=1 \text { (genuinely nonlinear), } j=1,3,
$$

where $c=\sqrt{p_{\rho}\left(\rho, S^{*}\right)}$. From (2.23) and (2.25), we have

$$
\lambda_{3}-\lambda_{2}=\lambda_{2}-\lambda_{1}=\sqrt{p_{\rho}\left(\rho, S^{*}\right)} \rightarrow 0, \quad \rho \rightarrow 0 .
$$

Therefore, system (2.20) is strictly hyperbolic in the nonvacuum states $\left\{\left(\rho, v, S^{*}\right) \mid \rho>\right.$ $0\}$, and the strict hyperbolicity fails near the vacuum states $\left\{\left(\rho, m / \rho, S^{*}\right)|| m / \rho \mid \leq\right.$ $\left.C_{0}, \rho=0\right\}$.

Now we consider the Riemann problem (2.20) and (2.3) for $U=(\rho, m, E)$ with constants $\rho_{ \pm} \geq 0, m_{ \pm}$, and $E_{ \pm}$satisfying $\left|m_{ \pm} / \rho_{ \pm}\right| \leq C_{0}<\infty$.

Given a state $U_{0}=\left(\rho_{0}, m_{0}, E_{0}\right)$, we consider possible states $U=(\rho, m, E)$ that can be connected to state $U_{0}$ on the right by a centered rarefaction wave in the $j$ families, $j=1,3$. Consider the self-similar solutions $(\rho, m, E)(\xi), \xi=x / t$, of the Riemann problem (2.20) and (2.3). Then we have

$$
\left\{\begin{array}{l}
\xi=\lambda_{j}(\rho, m, E)(\xi), \quad j=1,3 \\
\frac{d m}{d \xi}-\xi \frac{d \rho}{d \xi}=0 \\
\frac{d E}{d \xi}-\left(\frac{m}{\rho} \lambda_{j}-\frac{1}{2} \frac{m^{2}}{\rho^{2}}+\frac{p+\rho e}{\rho}\right) \frac{d \rho}{d \xi}=0
\end{array}\right.
$$


and, on the $j$-family centered rarefaction waves,

$$
\frac{\partial U}{\partial x}=\frac{1}{t} \frac{d U}{d \xi}=\frac{1}{t} r_{j}\left(U\left(\frac{x}{t}\right)\right), \quad j=1,3 .
$$

If $S_{-}^{*}=S_{+}^{*}$, then, for the Riemann problem (2.20), (2.23), and (2.3) satisfying $w_{j}\left(U_{+}\right) \geq w_{j}\left(U_{-}\right), j=1,3$, there exists a unique piecewise smooth entropy solution $R(x / t)$ containing the vacuum states on the upper half-plane $t>0$ and satisfying

$$
\left\{\begin{array}{l}
w_{1}(R(x / t)) \leq w_{1}\left(U_{+}\right), w_{3}(R(x / t)) \geq w_{3}\left(U_{-}\right) \\
w_{1}(R(x / t))-w_{3}(R(x / t)) \geq 0, S^{*}(x / t)=S^{*}{ }_{ \pm}
\end{array}\right.
$$

where $w_{j}=\frac{m}{\rho}+(-1)^{\frac{j-1}{2}} \int_{0}^{\rho} \frac{\sqrt{p_{\rho}\left(s, S^{*}\right)}}{s} d s, j=1,3$. These Riemann solutions are identical to those for the isentropic case with the 2 -family in the isentropic case corresponding to the 3-family in the nonisentropic case for the fixed constant $S^{*}$.

If $S^{*}-\neq S^{*}+$, then the Riemann solutions contain an additional contact discontinuity corresponding to the 2 -family, besides the rarefaction waves corresponding to the $\mathrm{j}$-families, $j=1,3$.

2.3. Divergence-Measure Vector Fields in $L^{\infty}$. We now briefly review the definition of normal traces in [5] and some properties of divergence-measure vector fields in $L^{\infty}$, especially the Gauss-Green formula, which will be used in Sections 3-4.

Definition 2.1. Let $D \subset \mathbb{R}^{N}$ be an open set. We say $F \in L^{\infty}\left(D ; \mathbb{R}^{N}\right)$ is a divergence-measure vector field if

$$
|\operatorname{div} F|(D)=\sup \left\{\int_{D} F \cdot \nabla \phi d y\left|\phi \in C_{0}^{1}(D ; \mathbb{R}),\right| \phi(y) \mid \leq 1, y \in D\right\}<\infty,
$$

which means that $\operatorname{div} F$ is a Radon measure over $D$. We define $\mathcal{D} M(D)$ as the space of divergence-measure vector fields over $D$ and, under the norm $\|F\|_{\mathcal{D} M}=$ $\|F\|_{L^{\infty}}+|\operatorname{div} F|(D), \mathcal{D} M(D)$ is a Banach space.

The relation between divergence-measure vector fields and hyperbolic conservation laws can be seen via the Lax entropy inequalities. For any $L^{\infty}$ entropy solution $U$ of (2.1)-(2.2), we deduce from the Lax entropy inequality (2.5) for entropy pair $(\eta, q)$ of $(2.1)$ and the Schwartz lemma [41] that

$$
\operatorname{div}_{(x, t)}(q(U(x, t)), \eta(U(x, t))) \quad \text { is a Radon measure in } \mathbb{R} \times(0, \infty),
$$

which implies $(q(U(x, t)), \eta(U(x, t))) \in \mathcal{D} M(\mathbb{R} \times(0, \infty))$.

One of the main points for divergence-measure vector fields is to understand the normal traces on deformable Lipschitz boundaries, since one cannot define the trace for each component of a $\mathcal{D} M$ field over any Lipschitz boundary in general, as opposed to the case of $B V$ vector fields. The notion of normal traces introduced in [5] has the advantage of providing essential information about the normal traces on certain hypersurfaces from the knowledge of the normal traces in its neighboring hypersurfaces, as we will see in Theorem 2.1. This advantage is made possible by introducing Lipschitz deformations, which are important not only for the definition of normal traces, but also for their applications. Note that a related notion of normal traces was also introduced with a different point of view in [2], in which a normal 
trace was defined as a representation function of a linear functional, in an abstract fashion. However, the Gauss-Green formula (2.32) (below) coincides, which means that both notions are consistent.

Let $\Omega$ be an open subset in $\mathbb{R}^{N}$. Following [5], we say that $\partial \Omega$ is a regular deformable Lipschitz boundary provided that the following hold.

(i) For each $\omega \in \partial \Omega$, there exist $r>0$ and a Lipschitz mapping $\gamma: \mathbb{R}^{N-1} \rightarrow \mathbb{R}$ such that, upon rotating and relabeling the coordinate axes if necessary,

$$
\Omega \cap Q(\omega, r)=\left\{y \in \mathbb{R}^{N} \mid \gamma\left(y_{1}, \cdots, y_{N-1}\right)<y_{N}\right\} \cap Q(\omega, r),
$$

where $Q(\omega, r)=\left\{y \in \mathbb{R}^{N}|| y_{i}-\omega_{i} \mid \leq r, i=1, \cdots, N\right\}$. We denote by $\tilde{\gamma}$ the $\operatorname{map}\left(y_{1}, \cdots, y_{N-1}\right) \mapsto\left(y_{1}, \cdots, y_{N-1}, \gamma\left(y_{1}, \cdots, y_{N-1}\right)\right)$.

(ii) There exists a map $\Psi: \partial \Omega \times[0,1] \rightarrow \bar{\Omega}$ such that $\Psi$ is a homeomorphism biLipschitz over its image and $\Psi(\cdot, 0) \equiv I$, where $I$ is the identity map over $\partial \Omega$. Denote $\partial \Omega_{\tau} \equiv \Psi(\partial \Omega \times\{\tau\}), \tau \in[0,1]$, and denote $\Omega_{\tau}$ the open subset of $\Omega$ whose boundary is $\partial \Omega_{\tau}$. We call $\Psi$ a Lipschitz deformation of $\partial \Omega$.

(iii) For a map $\tilde{\gamma}$ as in condition (i), and the map $\Psi_{\tau}$ of $\partial \Omega$ to $\partial \Omega_{\tau} \subset \Omega$, given by $\Psi_{\tau}(\omega)=\Psi(\omega, \tau), \lim _{\tau \rightarrow 0+} \nabla \Psi_{\tau} \circ \tilde{\gamma}=\nabla \tilde{\gamma}$ in $L_{l o c}^{1}(B)$, where $B$ denotes the largest open set such that $\tilde{\gamma}(B) \subset \partial \Omega$.

REMARK 2.1. Conditions (i)-(iii) above can be verified even for the star-shaped domains and the domains whose boundaries satisfy the cone property.

Let $F \in \mathcal{D} M(D)$. Let $\Omega \subset D$ be an open set with deformable Lipschitz boundary. Then, following [5], there exists $\mathcal{T} \subset(0,1)$ with meas $([0,1]-\mathcal{T})=0$ such that, for every $\tau \in \mathcal{T}$ and all $\phi \in C_{0}^{1}\left(\mathbb{R}^{N}\right)$,

$$
\left\langle\left.\operatorname{div} F\right|_{\Omega_{\tau}}, \phi\right\rangle=\int_{\partial \Omega_{\tau}} \phi(\omega) F(\omega) \cdot \nu_{\tau}(\omega) d \mathcal{H}^{N-1}(\omega)-\int_{\Omega_{\tau}} F(y) \cdot \nabla \phi(y) d y
$$

where $\nu_{\tau}$ is the unit outward normal field defined $\mathcal{H}^{N-1}$-almost everywhere in $\partial \Omega_{\tau}$.

THEOREM $2.2([5])$. (1). Let $F \in \mathcal{D} M(D)$ and $\Omega \subset D$ be an open set with regular deformable Lipschitz boundary. Then the limit

$$
\left.F \cdot \nu\right|_{\partial \Omega}=w-\lim _{\tau \rightarrow 0 \tau \in \mathcal{T}} F \cdot \nu_{\tau}, \quad \text { in } \mathcal{M}(\partial \Omega)
$$

exists when $F \cdot \nu_{\tau}$ are regarded as Radon measures on $\partial \Omega$ through the formula:

$$
\left\langle F \cdot \nu_{\tau}, \phi\right\rangle \equiv \int_{\partial \Omega_{\tau}} \phi\left(\Psi_{\tau}^{-1}(\omega)\right) F(\omega) \cdot \nu_{\tau}(\omega) d \mathcal{H}^{N-1}(\omega) .
$$

(2). This definition for $F \cdot \nu$ over $\partial \Omega$ yields the following Gauss-Green formula:

$$
\left\langle\left.\operatorname{div} F\right|_{\Omega}, \phi\right\rangle=\int_{\partial \Omega} \phi(\omega) F(\omega) \cdot \nu(\omega) d \mathcal{H}^{N-1}(\omega)-\int_{\Omega} F(y) \cdot \nabla \phi(y) d y
$$

for any $\phi \in C_{0}^{1}\left(\mathbb{R}^{N}\right)$, where the formal notation $F(\omega) \cdot \nu(\omega) d \mathcal{H}^{N-1}(\omega) \equiv F \cdot \nu$ is used for the normal trace measure justified in (3) below.

(3). The normal trace measure $F \cdot \nu$ has the following properties:

(i) $F \cdot \nu$ does not depend on the particular Lipschitz deformation for $\partial \Omega$ and is absolutely continuous with respect to $\left.\mathcal{H}^{N-1}\right|_{\partial \Omega}$; 
(ii) If $\partial \Omega \subset D$ and $|\operatorname{div} F|(\partial \Omega)=0$, the density of $F \cdot \nu$ coincides with the function $F \cdot \nu, \mathcal{H}^{N-1}$ - a.e. in $\partial \Omega$, whenever $\mathcal{H}^{N-1}(\partial \Omega \cap \mathcal{N})=0$, where $\mathcal{N}$ is the null set in the definition of the precise representative of $F$;

(iii) Denote also $F \cdot \nu$ the corresponding density. Then $F \cdot \nu \in L^{\infty}(\partial \Omega)$, and

$$
\begin{gathered}
\|F \cdot \nu\|_{L^{\infty}(\partial \Omega)} \leq\|F\|_{L^{\infty}(\Omega)}, \\
F \cdot \nu=w^{*}-\text { ess } \lim _{\tau \rightarrow 0+}\left(F \cdot \nu_{\tau}\right) \circ \Psi_{\tau}, \quad \text { in } L^{\infty}(\partial \Omega) .
\end{gathered}
$$

Furthermore, we have

Theorem $2.3([5])$. Let $g \in B V \cap L^{\infty}(D)$ and $F \in \mathcal{D} M(D)$. Then $g F \in$ $\mathcal{D} M(D)$. Moreover, if $g$ is Lipschitz continuous over any compact set in $D$, then $\operatorname{div}(g F)=g \operatorname{div} F+F \cdot \nabla g$.

For any Riemann solution $R(x / t)$ of $(2.1)$ and (2.3) and any $L^{\infty}$ entropy solution $U$ of (2.1)-(2.2) satisfying the Lax entropy inequality for a convex entropy pair $\left(\eta_{*}, q_{*}\right)$, we follow Dafermos $[14,17]$ to define

$$
\begin{aligned}
& \alpha(U, R)=\eta_{*}(U)-\eta_{*}(R)-\nabla \eta_{*}(R)(U-R), \\
& \beta(U, R)=q_{*}(U)-q_{*}(R)-\nabla \eta_{*}(R)(F(U)-F(R)) .
\end{aligned}
$$

Lemma 2.4. Assume that the Riemann solution $R(x / t)$ of (2.1) and (2.3) has bounded variation in $\mathbb{R}_{+}^{2} \equiv \mathbb{R} \times(0, \infty)$. Then

$$
\begin{gathered}
\left(q_{*}(U(x, t)), \eta_{*}(U(x, t))\right) \in \mathcal{D} M\left(\mathbb{R}_{+}^{2}\right), \\
(\beta(U(x, t), R(x / t)), \alpha(U(x, t), R(x / t))) \in \mathcal{D} M\left(\mathbb{R}_{+}^{2}\right) .
\end{gathered}
$$

This can be seen as follows. Define

$$
\mu=\partial_{t} \eta_{*}(U(x, t))+\partial_{x} q_{*}(U(x, t)), \quad d=\partial_{t} \alpha(U(x, t), R(x / t))+\partial_{x} \beta(U(x, t), R(x / t)) .
$$

Since $U(x, t)$ is an entropy solution, $\mu \leq 0$, and $d \leq 0$ in any region in which $R$ is constant, in the sense of distributions. The fact that $\mu$ and $d$ are Radon measures is a corollary of the Schwartz lemma [41] and the product rule in Theorem 2.3.

3. Stability of Rarefaction Waves for the Isentropic Euler Equations. In this section we show the global stability of rarefaction waves in the following class of weak entropy solutions of (2.6) and (2.2) containing the vacuum states.

DeFinition 3.1. A bounded measurable function $U(x, t)=(\rho(x, t), m(x, t))$ is an entropy solution of (2.6)-(2.8) and (2.2) in $\mathbb{R}_{+}^{2}$, if $U(x, t)$ satisfies the following.

(i). There exists $C>0$ such that

$$
0 \leq \rho(x, t) \leq C, \quad|m(x, t) / \rho(x, t)| \leq C .
$$

(ii). Equations (2.6) and initial data (2.2) are satisfied in the weak sense in $\mathbb{R}_{+}^{2}$, i.e., for all $\phi \in C_{0}^{1}\left(\mathbb{R}_{+}^{2}\right)$,

$$
\int_{0}^{\infty} \int_{-\infty}^{\infty}\left\{U \partial_{t} \phi+F(U) \partial_{x} \phi\right\} d x d t+\int_{-\infty}^{\infty} U_{0}(x) \phi(x, 0) d x=0
$$

with $U=(\rho, m)$ and $F(U)=\left(m, m^{2} / \rho+p(\rho)\right)$. 
(iii). The entropy inequality holds in the sense of distributions in $\mathbb{R}_{+}^{2}$, i.e., for any nonnegative function $\phi \in C_{0}^{1}\left(\mathbb{R}_{+}^{2}\right)$,

$$
\int_{0}^{\infty} \int_{-\infty}^{\infty}\left(\eta_{*}(U) \partial_{t} \phi+q_{*}(U) \partial_{x} \phi\right) d x d t+\int_{-\infty}^{\infty} \eta_{*}\left(U_{0}\right)(x) \phi(x, 0) d x \geq 0
$$

where $\left(\eta_{*}, q_{*}\right)$ is the mechanical energy-energy flux pair:

$$
\eta_{*}(U)=\frac{1}{2} \frac{m^{2}}{\rho}+\rho \int_{0}^{\rho} \frac{p(\sigma)}{\sigma^{2}} d \sigma, \quad q_{*}(U)=\frac{1}{2} \frac{m^{3}}{\rho^{2}}+m \int_{0}^{\rho} \frac{p(\sigma)}{\sigma^{2}} d \sigma+\frac{m p(\rho)}{\rho}
$$

REMARK 3.1. In Definition 3.1, we require that the entropy solutions in $L^{\infty}$ satisfy only one physical entropy inequality, a more broad class than the usual class of entropy solutions in $L^{\infty}$ that satisfy all weak Lax entropy inequalities.

REMARK 3.2. For the Cauchy problem (2.6)-(2.7) and (2.2) satisfying

$$
p(\rho)=\kappa \rho^{\gamma}(1+P(\rho)), \quad\left|P^{(k)}(\rho)\right| \leq C \rho^{1-k}, \quad 0 \leq k \leq 4, \quad \text { as } \rho \ll 1,
$$

the existence of global entropy solutions satisfying all weak Lax entropy inequalities has been established in Chen-LeFloch [11]. Also see DiPerna [18], Ding-Chen-Luo [16], Chen [4], Lions-Perthame-Tadmor [28], and Lions-Perthame-Souganidis [29] for the $\gamma$-law case.

THEOREM 3.2. Let $R(x / t)$ be the Riemann solution of (2.6)-(2.8) and (2.3), consisting of one or two rarefaction waves, constant states, and possible vacuum states, as constructed in §2.1. Let $U(x, t)$ be any entropy solution of (2.6)-(2.8) and (2.2) in $\mathbb{R}_{+}^{2}$, defined by Definition 3.1. Then, for any $L>0$,

$$
\int_{|x| \leq L} \alpha(U, R)(x, t) d x \leq \int_{|x| \leq L+M t} \alpha\left(U_{0}, R_{0}\right)(x) d x
$$

where $M>0$ depends only on $C>0$ in Definition 3.1 and is independent of $t$, and

$$
\begin{array}{r}
\alpha(U, R) \equiv(U-R)^{\top}\left(\int_{0}^{1} \nabla^{2} \eta_{*}(R+r(U-R)) d r\right)(U-R)>0, \\
\text { if } U \neq R \text { and both are away from the vacuum. }
\end{array}
$$

In particular, if $U_{0}(x)=R_{0}(x)$, then $U(x, t)=R(x / t)$ a.e.

Proof. Without loss of generality, we prove only for the Riemann solution (2.18) that consists of two rarefaction waves with the vacuum states as intermediate states. The other cases can be shown even simpler. The proof is based on the normal traces and Gauss-Green formula in Theorem 2.1 in $\S 2.3$ for divergence-measure vector fields and the techniques developed in $[6,7,17]$ for strictly hyperbolic systems. One of the new difficulties here is that the strict hyperbolicity fails near the vacuum, which yields the singularity of the derivatives of the mechanical energy near the vacuum, and the integrability or boundedness of certain quantities related to the mechanical energy must be carefully analyzed near the vacuum, which is naturally ensured in the strictly hyperbolic case. Another difficulty is that the entropy solutions are only in $L^{\infty}$. 
1. Denote $U=(\rho, m)$ and $R=(\bar{\rho}, \bar{m})$. First we renormalize the mechanical energy-energy flux pair $\left(\eta_{*}, q_{*}\right)$, as defined in (2.35)-(2.36), and consider

$$
\mu=\partial_{t} \eta_{*}(U(x, t))+\partial_{x} q_{*}(U(x, t)), d=\partial_{t} \alpha(U(x, t), R(x / t))+\partial_{x} \beta(U(x, t), R(x / t)) .
$$

Since $U(x, t)$ is an entropy solution, $\mu \leq 0$, and $d \leq 0$ in any region in which $R$ is constant, in the sense of distributions. Then Lemma 2.1 implies that $\mu$ and $d$ are Radon measures, and

$$
\begin{gathered}
\left(q_{*}(U(x, t)), \eta_{*}(U(x, t))\right) \in \mathcal{D} M\left(\mathbb{R}_{+}^{2}\right), \\
(\beta(U(x, t), R(x / t)), \alpha(U(x, t), R(x / t))) \in \mathcal{D} M\left(\mathbb{R}_{+}^{2}\right) .
\end{gathered}
$$

Set

$\Omega_{1}=\left\{(x, t) \mid \lambda_{1}\left(U_{-}\right)<x / t<v_{c_{1}}, t>0\right\}, \Omega_{2}=\left\{(x, t) \mid v_{c_{2}}<x / t<\lambda_{2}\left(U_{+}\right), t>0\right\}$, the rarefaction wave regions of the Riemann solution. Over these regions,

$$
d=\partial_{t} \alpha(U, R)+\partial_{x} \beta(U, R)=\mu-\left(\partial_{x} R\right)^{\top} \nabla^{2} \eta_{*}(R) Q F(U, R),
$$

where $Q F(U, R)=F(U)-F(R)-\nabla F(R)(U-R)$, and we used the fact that $\nabla^{2} \eta_{*} \nabla F$ is symmetric. Recall that, for $(x, t) \in \Omega_{j}$,

$$
\frac{\partial R(x / t)}{\partial x}=\frac{1}{t} r_{j}(R(x / t)), \quad j=1,2 .
$$

Then, by (3.6) and (3.7), for any Borel set $E \subset \Omega_{j}, j=1,2$, we have

$$
d(E)=\mu(E)-\int_{E} \frac{1}{t} r_{j}(R)^{\top} \nabla^{2} \eta_{*}(R) Q F(U, R)(x, t) d x d t .
$$

2. For any $L>0$, let $\Pi_{L, t}^{\delta}$ denote the region $\{(x, s)|| x \mid<L+M(t-s), 0<\delta<$ $s<t\}$ and $\Omega_{j}^{\delta}(t)=\Omega_{j} \cap \Pi_{L, t}^{\delta}, \Omega_{j}(t)=\Omega_{j} \cap\{(x, s) \mid 0<s<t\}, j=1,2$, where

$$
M \geq M_{0} \equiv\|\beta(U, R) / \alpha(U, R)\|_{L^{\infty}\left(\mathbb{R}_{+}^{2}\right)} .
$$

First, by the entropy inequality (3.2), the normal traces and Gauss-Green formula (Theorem 2.1) for divergence-measure vector fields, and the convexity of $\eta_{*}(U)$ in $U$, it is standard (cf. [12]) to deduce that any entropy solution defined in Definition 3.1 assumes its initial data $U_{0}(x)$ strongly in $L_{l o c}^{1}$ :

$$
\lim _{t \rightarrow 0} \int_{|x| \leq K}\left|U(x, t)-U_{0}(x)\right| d x=0, \quad \text { for any } K>0 .
$$

Furthermore, we apply Theorem 2.1 again to conclude

$$
\begin{aligned}
d\left\{\Pi_{t, L}^{\delta}\right\}= & \int_{|x| \leq L} \alpha(U(x, t), R(x / t)) d x-\int_{|x| \leq L+M(t-\delta)} \alpha(U(x, \delta), R(x / \delta)) d x \\
& +\int_{\partial \Pi_{t, L}^{\delta}}(\beta, \alpha) \cdot \nu d \sigma
\end{aligned}
$$


where $\nu$ is the unit outward normal field, and $\sigma$ is the boundary measure. Then we can choose $M \geq M_{0}$ such that $\int_{\partial \Pi_{t, L}^{\delta}}(\beta, \alpha) \cdot \nu d \sigma \geq 0$. Therefore, we have

$$
\begin{aligned}
d\left\{\Pi_{t, L}^{\delta}\right\} \geq & \int_{|x| \leq L} \alpha(U(x, t), R(x / t)) d x \\
& -\int_{|x| \leq L+M(t-\delta)} \alpha(U(x, \delta), R(x / \delta)) d x .
\end{aligned}
$$

On the other hand, from (3.8), we have

$$
d\left\{\Omega_{j}^{\delta}(t)\right\}=\mu\left\{\Omega_{j}^{\delta}(t)\right\}-\sum_{j=1}^{2} \int_{\Omega_{j}^{\delta}(t)} \frac{1}{s} r_{j}(R)^{\top} \nabla^{2} \eta_{*}(R) Q F(U, R)(x, s) d x d s .
$$

Since $R(x / t)$ is constant in each component of $\Pi_{t}-\left\{\Omega_{1}^{\delta}(t) \cup \Omega_{2}^{\delta}(t)\right\}$ and $\mu \leq 0$, we have

$$
d\left\{\Pi_{t, L}^{\delta}\right\} \leq-\sum_{j=1}^{2} \int_{\Omega_{j}^{\delta}(t)} \frac{1}{s} r_{j}(R)^{\top} \nabla^{2} \eta_{*}(R) Q F(U, R)(x, s) d x d s .
$$

3. We now prove that $h_{j}(x, s) \equiv r_{j}(R)^{\top} \nabla^{2} \eta_{*}(R) Q F(U, R)(x, s), j=1,2$, are nonnegative and uniformly bounded in $\bar{\Omega}_{1}(t) \cup \bar{\Omega}_{2}(t)$ as $s>0$. A careful direct calculation yields

$$
r_{j}(R)^{\top} \nabla^{2} \eta_{*}(R)=\frac{2 p^{\prime}(\bar{\rho})}{\bar{\rho} p^{\prime \prime}(\bar{\rho})+2 p^{\prime}(\bar{\rho})}\left(-\frac{\bar{m}}{\bar{\rho}}+(-1)^{j} \sqrt{p^{\prime}(\bar{\rho})}, 1\right)
$$

and

$$
Q F(U, R)=\left(\begin{array}{c}
0 \\
\rho\left(\frac{m}{\rho}-\frac{\bar{m}}{\bar{\rho}}\right)^{2}+p-\bar{p}-\bar{p}_{\rho}(\rho-\bar{\rho})
\end{array}\right) .
$$

Therefore, we have

$$
h_{j}(x, s)=\frac{2 p^{\prime}(\bar{\rho})}{\bar{\rho} p^{\prime \prime}(\bar{\rho})+2 p^{\prime}(\bar{\rho})}\left(\rho\left(\frac{m}{\rho}-\frac{\bar{m}}{\bar{\rho}}\right)^{2}+p(\rho)-p(\bar{\rho})-p^{\prime}(\bar{\rho})(\rho-\bar{\rho})\right) \geq 0,
$$

since $p(\rho)$ is convex in $\rho \geq 0$. Also, from (2.7)-(2.8), we can see that $h_{j}(x, s), j=1,2$, are indeed uniformly bounded everywhere even at the vacuum states, which means that $h_{j}(x, s), j=1,2$, are integrable in $\Omega_{1}(t) \cup \Omega_{2}(t)$ as $s>0$.

This fact in combination with (3.10) yields

$$
\int_{|x| \leq L} \alpha(U(x, t), R(x / t)) d x \leq \int_{|x| \leq L+M(t-\delta)} \alpha(U(x, \delta), R(x / \delta)) d x .
$$

Then (3.9) and (3.12) imply (3.5) as $\delta \rightarrow 0$. This completes the proof.

In the previous proof, the values of the divergence-measure fields $(\beta(U, R), \alpha(U, R))(x, t)$ on the line segments in the $(x, t)$-plane should be understood in the sense of the normal traces. To recover the classical sense, result (3.5) should be stated that (3.5) holds for a.e. $t \in[0, \infty)$.

As a corollary, we conclude 
THEOREM 3.3. Let $R(x / t)$ be the Riemann solution of (2.6)-(2.8) and (2.3), consisting of one or two rarefaction waves, constant states, and possible vacuum states, as constructed in §2.1. Let $U(x, t)$ be any entropy solution of (2.6)-(2.8), (2.2), and (2.4), defined by Definition 3.1. Then $U(x, t)$ is asymptotically stable under the initial perturbation $P_{0}(x) \in L^{1} \cap L^{\infty}$ in the sense of

$$
\lim _{t \rightarrow \infty} \int_{|\xi| \leq L} \alpha(U(\xi t, t), R(\xi)) d \xi=0, \quad \text { for any } L>0 .
$$

4. Stability of Rarefaction Waves for the Non-Isentropic Euler Equations. In this section we show the global stability of rarefaction waves in the following class of weak entropy solutions of (2.20)-(2.22) and (2.2) containing the vacuum states, even for non-isentropic fluids.

Definition 4.1. A bounded measurable function $U(x, t)=(\rho(x, t), m(x, t), E(x, t))$ is an entropy solution of (2.20)-(2.22) and (2.2) in $\mathbb{R}_{+}^{2}$, if it satisfies the following.

(i). There exists $C>0$ such that

$$
0 \leq \rho(x, t) \leq C, \quad|m(x, t) / \rho(x, t)| \leq C, \quad 0<\underline{S}_{0}^{*} \leq S^{*}(x, t) \leq \bar{S}_{0}^{*}<\infty .
$$

(ii). Equations (2.20) and initial data (2.2) are satisfied in the weak sense in $\mathbb{R}_{+}^{2}$, i.e., for all $\phi \in C_{0}^{1}\left(\mathbb{R}^{2}\right)$,

$$
\int_{0}^{\infty} \int_{-\infty}^{\infty}\left\{U \partial_{t} \phi+F(U) \partial_{x} \phi\right\} d x d t+\int_{-\infty}^{\infty} U_{0}(x) \phi(x, 0) d x=0,
$$

with $U=(\rho, m, E)$ and $F(U)=\left(m, \frac{m^{2}}{\rho}+p, m\left(\frac{1}{2} \frac{m^{2}}{\rho^{2}}+e+\frac{p}{\rho}\right)\right)$.

(iii). The Clausius inequality holds in the sense of distributions in $\mathbb{R}_{+}^{2}$, i.e., for any nonnegative function $\phi \in C_{0}^{1}\left(\mathbb{R}^{2}\right)$,

$$
\int_{0}^{\infty} \int_{-\infty}^{\infty}\left(\rho \alpha\left(S^{*}\right) \partial_{t} \phi+m \alpha\left(S^{*}\right) \partial_{x} \phi\right) d x d t+\int_{-\infty}^{\infty} \rho_{0}(x) \alpha\left(S_{0}^{*}(x)\right) \phi(x, 0) d x \leq 0,
$$

for any $C^{2}$ function $\alpha\left(S^{*}\right)$ with $\alpha^{\prime}\left(S^{*}\right) \geq 0$.

Choose $S=\alpha_{0}\left(S^{*}\right)$ with $\alpha_{0}^{\prime}\left(S^{*}\right)>0$ for $0<\underline{S}_{0}^{*} \leq S^{*} \leq \bar{S}_{0}^{*}<\infty$, to be determined later, such that, for $a=\alpha_{0}^{-1}$,

$$
S^{*}=a(S), \quad a^{\prime}(S)>0, \quad a^{\prime \prime}(S)>0,
$$

for $0<\underline{S}_{0} \equiv \alpha\left(\underline{S}_{0}^{*}\right) \leq S \leq \alpha\left(\bar{S}_{0}^{*}\right) \equiv \bar{S}_{0}<\infty$. Then

$$
p\left(\rho, S^{*}\right)=p(\rho, a(S)), e\left(\rho, S^{*}\right)=e(\rho, a(S)), \theta\left(\rho, S^{*}\right)=\theta(\rho, a(S)),
$$

and, by setting $H=\rho S$, the Clausius inequality becomes

$$
\partial_{t} H+\partial_{x}(m H / \rho) \geq 0
$$

in the sense of distributions.

We first require $\alpha_{0}\left(S^{*}\right)$ to satisfy

$$
0<\bar{S}_{0}-\underline{S}_{0} \ll 1 .
$$


Denote $W=(\rho, m, H)$ and $\mathcal{V}_{C}=\left\{W|0 \leq \rho \leq C|, m / \rho \mid \leq C, \underline{S}_{0} \leq S \leq \bar{S}_{0}\right\}$. Choose $\left(\eta_{*}, q_{*}\right)$ the mechanical energy-energy flux pair:

$$
\begin{aligned}
& \eta_{*}(W)=E \equiv \frac{1}{2} \frac{m^{2}}{\rho}+\rho e\left(\rho, a\left(\frac{H}{\rho}\right)\right), \\
& q_{*}(W)=\frac{m}{\rho}(E+p) \equiv \frac{1}{2} \frac{m^{3}}{\rho^{2}}+m e\left(\rho, a\left(\frac{H}{\rho}\right)\right)+\frac{m p\left(\rho, a\left(\frac{H}{\rho}\right)\right)}{\rho} .
\end{aligned}
$$

Then the conservation of energy implies $\partial_{t} \eta_{*}(W)+\partial_{x} q_{*}(W)=0$ in the sense of distributions.

Since $a^{\prime}(S)>0$, one has

$$
\partial_{H} \eta_{*}(W)=a^{\prime}(S) \theta(\rho, a(S)) \geq 0
$$

We choose $a(S) \in C^{2}$ such that, for $W \in \mathcal{V}_{C}$,

$$
a^{\prime \prime}(S)>a^{\prime}(S)^{2}\left(\frac{p_{a}^{2}}{\rho^{2} p_{\rho} \theta}-\frac{\theta_{a}}{\theta}\right)
$$

which implies the strict convexity of $\eta_{*}(W)$ for $W \in \mathcal{V}_{C}$ :

$$
\nabla_{W}^{2} \eta_{*}(W)>0, \quad W \in \mathcal{V}_{C}
$$

THEOREM 4.2. Let $R(x / t)$ be the Riemann solution of (2.20), (2.23), and (2.3) consisting of one or two rarefaction waves, constant states, and possible vacuum states with $S^{*} \equiv \bar{S}^{*}=$ const., as constructed in §2.2. Let $U(x, t)$ be any entropy solution of (2.20), (2.23), and (2.2) in $\mathbb{R}_{+}^{2}$, defined by Definition 4.1, such that

$$
S^{*}(x, t) \geq \bar{S}^{*}
$$

Then, for any $L>0$,

$$
\int_{|x| \leq L} \alpha(W, \bar{W})(x, t) d x \leq \int_{|x| \leq L+M t} \alpha\left(W_{0}, \bar{W}_{0}\right)(x) d x
$$

where $M>0$ depends only on $\mathcal{V}_{C}$, independent of $t$; and $W(x, t)=(\rho, m, H)(x, t)$ corresponds to the entropy solution $U, \bar{W}(x, t)=(\bar{\rho}, \bar{m}, \bar{H})(x / t)$ corresponds to the Riemann solution $R(x / t)$, and

$$
\begin{gathered}
\alpha(W, \bar{W})=(W-\bar{W})^{\top}\left(\int_{0}^{1} \nabla^{2} \eta_{*}(\bar{W}+r(W-\bar{W})) d r\right)(W-\bar{W})>0 \\
\text { when } W \neq \bar{W} \text { and both are away from the vacuum. }
\end{gathered}
$$

In particular, if $U_{0}(x)=R_{0}(x)$, then $U(x, t)=R(x / t)$ a.e.

Proof. Without loss of generality, we prove only for the Riemann solution that consists of two rarefaction waves with the vacuum states as intermediate states, as constructed in $\S 2.2$. The other cases can be shown similarly. Since the essential effect of the physical entropy $S^{*}$ for the non-isentropic case, the approach for the isentropic Euler equations can not directly work for the non-isentropic case. The approach for the system away from the vacuum as in [6] also fails if one chooses the typical physical entropy $S^{*}$ as an independent thermodynamical variable. The main idea to overcome 
this difficulty is to choose an appropriate function of $S^{*}$ as a new variable and work on the state variables $W$ instead of the original variables $U$, in combination with the techniques in $\S 3$ and [6].

1. First we renormalize the mechanical energy-energy flux pair $\left(\eta_{*}, q_{*}\right)$ with respect to $W$ and $\bar{W}$ (not $U$ and $R$ !) as in (2.35)-(2.36) to have

$$
\begin{aligned}
& \alpha(W, \bar{W})=\eta_{*}(W)-\eta_{*}(\bar{W})-\nabla \eta_{*}(\bar{W})(W-\bar{W}) \geq 0, \\
& \beta(W, \bar{W})=q_{*}(W)-q_{*}(\bar{W})-\nabla \eta_{*}(\bar{W})(\tilde{F}(W)-\tilde{F}(\bar{W})),
\end{aligned}
$$

where $\tilde{F}(W)=\left(m, m^{2} / \rho+p, m H / \rho\right)^{\top}$, and we have used (4.11). Then we consider

$$
\begin{aligned}
& \mu=-\partial_{H} \eta_{*}(\bar{W}(x / t))\left(\partial_{t} H+\partial_{x}(m H / \rho)\right)(x, t), \\
& d=\partial_{t} \alpha(W(x, t), \bar{W}(x / t))+\partial_{x} \beta(W(x, t), \bar{W}(x / t)) .
\end{aligned}
$$

Since $U(x, t)$ is an entropy solution, (4.5) and (4.9) yield $\mu \leq 0$ in the sense of distributions, and, in any region in which $\bar{W}$ is constant, $d=\mu \leq 0$. Lemma 2.1 implies that $\mu$ and $d$ are Radon measures, and $(\beta(W(x, t), \bar{W}(x / t)), \alpha(W(x, t), \bar{W}(x / t))) \in$ $\mathcal{D} M\left(\mathbb{R}_{+}^{2}\right)$.

Set

$\Omega_{1}=\left\{(x, t) \mid \lambda_{1}\left(U_{-}\right)<x / t<v_{c_{1}}, t>0\right\}, \Omega_{3}=\left\{(x, t) \mid v_{c_{2}}<x / t<\lambda_{3}\left(U_{+}\right), t>0\right\}$, the rarefaction wave regions of the Riemann solution. Over these regions,

$$
d=\partial_{t} \alpha(W, \bar{W})+\partial_{x} \beta(W, \bar{W})=\mu-\left(\partial_{x} \bar{W}\right)^{\top} \nabla^{2} \eta_{*}(\bar{W}) Q \tilde{F}(W, \bar{W}),
$$

where $Q \tilde{F}(W, \bar{W})=\tilde{F}(W)-\tilde{F}(\bar{W})-\nabla \tilde{F}(\bar{W})(W-\bar{W})$, and we used the fact that $\nabla^{2} \eta_{*} \nabla \tilde{F}$ is symmetric.

2. A careful calculation indicates that, for $(x, t) \in \Omega_{j}$,

$$
\frac{\partial \bar{W}(x / t)}{\partial x}=\frac{1}{t} \hat{r}_{j}(\bar{W}(x / t)), \quad j=1,3
$$

where

$$
\hat{r}_{j}(\bar{W}(x / t))=\alpha_{j}\left(1, \bar{m} / \bar{\rho}+(-1)^{\frac{j+1}{2}} \sqrt{\bar{p}_{\rho}}, \bar{S}\right)^{\top}, \quad \alpha_{j}=(-1)^{\frac{j+1}{2}} \frac{2 \bar{\rho} \sqrt{\bar{p}_{\rho}}}{\bar{\rho} \bar{p}_{\rho \rho}+2 \bar{p}_{\rho}} .
$$

Then, by (4.19) and (4.20), for any Borel set $E \subset \Omega_{j}, j=1,3$, we have

$$
d(E)=\mu(E)-\int_{E} \frac{1}{t} \hat{r}_{j}(\bar{W})^{\top} \nabla^{2} \eta_{*}(\bar{W}) Q \tilde{F}(W, \bar{W})(x, t) d x d t .
$$

3. For any $L>0$, let $\Pi_{L, t}^{\delta}$ denote the region $\{(x, s)|| x \mid<L+M(t-s), 0<\delta<$ $s<t\}$ and $\Omega_{j}^{\delta}(t)=\Omega_{j} \cap \Pi_{L, t}^{\delta}, \Omega_{j}(t)=\Omega_{j} \cap\{(x, s) \mid 0<s<t\}, j=1,2$, where

$$
M \geq M_{0} \equiv\|\beta(W, \bar{W}) / \alpha(W, \bar{W})\|_{L^{\infty}\left(\mathbb{R}_{+}^{2}\right)} .
$$

First, by (4.1) and (4.11), the normal traces and Gauss-Green formula (Theorem 2.1) for divergence-measure vector fields, and the convexity of $\eta_{*}(W)$ in $W$, it is also standard (cf. [12]) to deduce that $W(x, t)$ assumes its initial data $W_{0}(x)$ strongly in $L_{\text {loc }}^{1}$ :

$$
\lim _{t \rightarrow 0} \int_{|x| \leq K}\left|W(x, t)-W_{0}(x)\right| d x=0, \quad \text { for any } K>0
$$


Furthermore, we apply Theorem 2.1 again to conclude

$$
\begin{aligned}
d\left\{\Pi_{t, L}^{\delta}\right\}= & \int_{|x| \leq L} \alpha(W(x, t), \bar{W}(x / t)) d x-\int_{|x| \leq L+M(t-\delta)} \alpha(W(x, \delta), \bar{W}(x / \delta)) d x \\
& +\int_{\partial \Pi_{t, L}^{\delta}}(\beta, \alpha) \cdot \nu d \sigma
\end{aligned}
$$

where $\nu$ is the unit outward normal field. Then we can choose $M \geq M_{0}$ such that

$$
\int_{\partial \Pi_{t, L}^{\delta}}(\beta, \alpha) \cdot \nu d \sigma \geq 0
$$

Therefore, we have

$$
\begin{aligned}
d\left\{\Pi_{t, L}^{\delta}\right\} \geq & \int_{|x| \leq L} \alpha(W(x, t), \bar{W}(x / t)) d x \\
& -\int_{|x| \leq L+M(t-\delta)} \alpha(W(x, \delta), \bar{W}(x / \delta)) d x .
\end{aligned}
$$

On the other hand, from (4.21), we have

$$
d\left\{\Omega_{j}^{\delta}(t)\right\}=\mu\left\{\Omega_{j}^{\delta}(t)\right\}-\sum_{j=1,3} \int_{\Omega_{j}^{\delta}(t)} \frac{1}{s} r_{j}(\bar{W})^{\top} \nabla^{2} \eta_{*}(\bar{W}) Q \tilde{F}(W, \bar{W})(x, s) d x d s .
$$

Since $R(x / t)$ is constant in each component of $\Pi_{t}-\left\{\Omega_{1}(t) \cup \Omega_{2}(t)\right\}$ and $\mu \leq 0$, we have

$$
d\left\{\Pi_{t, L}^{\delta}\right\} \leq-\sum_{j=1,3} \int_{\Omega_{j}^{\delta}(t)} \frac{1}{s} \hat{r}_{j}(\bar{W})^{\top} \nabla^{2} \eta_{*}(\bar{W}) Q \tilde{F}(W, \bar{W})(x, s) d x d s .
$$

4. We now prove that

$$
h_{j}(x, s) \equiv \hat{r}_{j}(\bar{W})^{\top} \nabla^{2} \eta_{*}(\bar{W}) Q \tilde{F}(W, \bar{W})(x, s), \quad j=1,3,
$$

are nonnegative and uniformly bounded in $\bar{\Omega}_{1}(t) \cup \bar{\Omega}_{3}(t)$ as $s>0$. By a direct calculation, we have

$$
Q \tilde{F}(W, \bar{W})=\left(\begin{array}{c}
0 \\
\rho\left(\frac{m}{\rho}-\frac{\bar{m}}{\bar{\rho}}\right)^{2}+p-\bar{p}-\bar{p}_{\rho}(\rho-\bar{\rho})-\frac{\rho}{\bar{\rho}} \bar{p}_{a} \bar{a}^{\prime}(S-\bar{S}) \\
\rho\left(\frac{m}{\rho}-\frac{\bar{m}}{\bar{\rho}}\right)(S-\bar{S})
\end{array}\right)
$$

and

$$
\hat{r}_{j}(W)^{\top} \nabla^{2} \eta_{*}(W)=\frac{\alpha_{j}}{\rho}\left(p_{\rho}+(-1)^{\frac{j-1}{2}} \frac{m \sqrt{p_{\rho}}}{\rho}-\frac{p_{a} a^{\prime} S}{\rho},(-1)^{\frac{j+1}{2}} c, \frac{p_{a} a^{\prime}}{\rho}\right)
$$

Therefore, we have

$$
h_{j}(x, s)=\frac{2 \bar{p}_{\rho}}{\bar{\rho} \bar{p}_{\rho \rho}+2 \bar{p}_{\rho}} \Delta_{j}(W, \bar{W})(x, s)
$$


where

$$
\begin{aligned}
\Delta_{j}(W, \bar{W})= & \rho\left(\frac{m}{\rho}-\frac{\bar{m}}{\bar{\rho}}\right)^{2}+(-1)^{\frac{j+1}{2}} \frac{\bar{p}_{a} \bar{a}^{\prime}}{\bar{\rho} \sqrt{\bar{p}}_{\rho}} \rho\left(\frac{m}{\rho}-\frac{\bar{m}}{\bar{\rho}}\right)(S-\bar{S}) \\
& +p-\bar{p}-\bar{p}_{\rho}(\rho-\bar{\rho})-\bar{p}_{a} \bar{a}^{\prime}(S-\bar{S})-\frac{\bar{p}_{a} \bar{a}^{\prime}}{\bar{\rho}}(\rho-\bar{\rho})(S-\bar{S}) \\
= & p-\bar{p}-\bar{p}_{\rho}(\rho-\bar{\rho})-\bar{p}_{a} \bar{a}^{\prime}(S-\bar{S})-\frac{\bar{p}_{a} \bar{a}^{\prime}}{\bar{\rho}}(\rho-\bar{\rho})(S-\bar{S}) \\
& -\frac{\bar{p}_{a}^{2}\left(\bar{a}^{\prime}\right)^{2}}{4 \bar{\rho}^{2} \bar{p}_{\rho}} \rho(S-\bar{S})^{2}+\rho\left(\left(\frac{m}{\rho}-\frac{\bar{m}}{\bar{\rho}}\right)+(-1)^{\frac{j+1}{2}} \frac{\bar{p}_{a} \bar{a}^{\prime}}{2 \bar{\rho} \sqrt{\bar{p}_{\rho}}}(S-\bar{S})\right)^{2} \\
\geq & p-\bar{p}-\bar{p}_{\rho}(\rho-\bar{\rho})-\bar{p}_{a} \bar{a}^{\prime}(S-\bar{S}) \\
& -\frac{\bar{p}_{a} \bar{a}^{\prime}}{\bar{\rho}}(\rho-\bar{\rho})(S-\bar{S})-\frac{\bar{p}_{a}^{2}\left(\bar{a}^{\prime}\right)^{2}}{4 \bar{\rho}^{2} \bar{p}_{\rho}} \rho(S-\bar{S})^{2} .
\end{aligned}
$$

This means that there exists $(\hat{\rho}, \hat{S})$ such that

$$
2 \Delta_{j}(W, \bar{W}) \geq Q-\tilde{Q}
$$

where

$$
\begin{aligned}
Q= & \hat{p}_{\rho \rho}(\rho-\bar{\rho})^{2}+\left(\hat{p}_{a} a^{\prime \prime}(\hat{S})+a^{\prime}(\hat{S})^{2}\left(\hat{p}_{a a}-\frac{\hat{p}_{a}^{2}}{2 \hat{\rho} \hat{p}_{\rho}}\right)\right)(S-\bar{S})^{2} \\
& +2\left(\hat{p}_{\rho a}-\frac{\hat{p}_{a}}{\hat{\rho}}\right) a^{\prime}(\hat{S})(\rho-\bar{\rho})(S-\bar{S})
\end{aligned}
$$

and

$$
\begin{aligned}
\tilde{Q} & =\frac{1}{2}\left(\frac{\rho \bar{p}_{a}^{2} a^{\prime}(\bar{S})^{2}}{\bar{\rho}^{2} \bar{p}_{\rho}}-\frac{\hat{p}_{a}^{2} a^{\prime}(\hat{S})^{2}}{\hat{\rho} \hat{p}_{\rho}}\right)(S-\bar{S})^{2}+2\left|\frac{\hat{p}_{a} a^{\prime}(\hat{S})}{\hat{\rho}}-\frac{\bar{p}_{a} a^{\prime}(\bar{S})}{\bar{\rho}}\|\rho-\bar{\rho}\| S-\bar{S}\right| \\
& =\frac{\kappa}{2 \gamma c_{v}^{2}}\left(\bar{\rho}^{\gamma-1} \rho A(\bar{S})-\hat{\rho}^{\gamma} A(\hat{S})\right)(S-\bar{S})^{2}+\frac{2 \kappa}{c_{v}}\left|\hat{\rho}^{\gamma-1} B(\hat{S})-\bar{\rho}^{\gamma-1} B(\bar{S})\right||\rho-\bar{\rho} \| S-\bar{S}|,
\end{aligned}
$$

where $A(S)=a^{\prime}(S)^{2} e^{a(S) / c_{v}}$ and $B(S)=a^{\prime}(S) e^{a(S) / c_{v}}$ are increasing functions in $S$. Since $\bar{S} \leq \hat{S} \leq S$, we have

$$
\begin{aligned}
& \tilde{Q} \leq \frac{\kappa}{2 \gamma c_{v}^{2}} \hat{\rho}^{\gamma}(A(\bar{S})-A(\hat{S}))(S-\bar{S})^{2} \\
&+C|S-\bar{S}|\left\{A(\bar{S})\left|\hat{\rho}^{\gamma}-\bar{\rho}^{\gamma-1} \rho\right||S-\bar{S}|+\hat{\rho}^{\gamma-1}|B(\hat{S})-B(\bar{S})||\rho-\bar{\rho}|\right. \\
&\left.+B(\bar{S})\left|\hat{\rho}^{\gamma-1}-\bar{\rho}^{\gamma-1}\right||\rho-\bar{\rho}|\right\} \\
& \leq C|S-\bar{S}|\left\{a^{\prime}(\bar{S})^{2}\left|\hat{\rho}^{\gamma}-\bar{\rho}^{\gamma-1} \rho\right||S-\bar{S}|+B(\hat{S}) \hat{\rho}^{\gamma-1}|\rho-\bar{\rho}|\right. \\
& \\
&\left.\quad+a^{\prime}(\bar{S})|\rho-\bar{\rho}|\left|\hat{\rho}^{\gamma-1}-\bar{\rho}^{\gamma-1}\right|\right\} .
\end{aligned}
$$

When $\bar{\rho} \leq \hat{\rho} \leq \rho$, we obtain

$$
\begin{aligned}
& \left|\hat{\rho}^{\gamma-1}-\bar{\rho}^{\gamma-1}\right| \leq \hat{\rho}^{-1}\left(\left|\hat{\rho}^{\gamma}-\bar{\rho}^{\gamma}\right|+\bar{\rho}^{\gamma-1}|\hat{\rho}-\bar{\rho}|\right) \leq C \hat{\rho}^{\gamma-2}|\rho-\bar{\rho}|, \\
& \left|\hat{\rho}^{\gamma}-\bar{\rho}^{\gamma-1} \rho\right| \leq \bar{\rho}^{\gamma-1}|\rho-\bar{\rho}|+\left|\hat{\rho}^{\gamma}-\bar{\rho}^{\gamma}\right| \leq\left(\hat{\rho}^{\gamma-1}+\gamma \hat{\rho}^{\gamma-1}\right)|\rho-\bar{\rho}| \leq C \hat{\rho}^{\gamma-1}|\rho-\bar{\rho}|,
\end{aligned}
$$


which implies from (4.32) that

$$
\begin{aligned}
\tilde{Q} \leq C\{ & a^{\prime}(\bar{S})^{2} \hat{\rho}^{\gamma-1}|\rho-\bar{\rho}||S-\bar{S}|^{2}+a^{\prime}(\hat{S}) \hat{\rho}^{\gamma-1}|\rho-\bar{\rho}||S-\bar{S}| \\
& \left.+a^{\prime}(\bar{S}) \hat{\rho}^{\gamma-2}|\rho-\bar{\rho}|^{2}|S-\bar{S}|\right\} .
\end{aligned}
$$

When $\rho \leq \hat{\rho} \leq \bar{\rho}$, we use $\gamma \in(1,2]$ and the mean value theorem to have

$$
\begin{aligned}
& \left|\hat{\rho}^{\gamma-1}-\bar{\rho}^{\gamma-1}\right|=(\gamma-1) \tilde{\rho}^{\gamma-2}|\hat{\rho}-\bar{\rho}| \leq(\gamma-1) \hat{\rho}^{\gamma-2}|\rho-\bar{\rho}|, \\
& \left|\hat{\rho}^{\gamma}-\bar{\rho}^{\gamma-1} \rho\right| \leq \hat{\rho}^{\gamma-1}|\hat{\rho}-\rho|+\hat{\rho}\left|\hat{\rho}^{\gamma-1}-\bar{\rho}^{\gamma-1}\right| \leq C \hat{\rho}^{\gamma-1}|\rho-\bar{\rho}|,
\end{aligned}
$$

which also implies (4.33) from (4.32).

On the other hand, we choose $a(S)$ such that

$$
a^{\prime \prime}(S) \geq N a^{\prime}(S)^{2},
$$

with

$$
N>\max \left\{\sup _{\mathcal{V}_{C}}\left(\frac{p_{a}^{2}}{\rho^{2} p_{\rho} \theta}-\frac{\theta_{a}}{\theta}\right), \sup _{\mathcal{V}_{C}}\left(\frac{1}{\hat{p}_{a} \hat{p}_{\rho \rho}}\left(\left(\hat{p}_{\rho a}-\frac{\hat{p}_{a}}{\hat{\rho}}\right)^{2}-\hat{p}_{\rho \rho}\left(\hat{p}_{a a}-\frac{\hat{p}_{a}^{2}}{2 \hat{\rho} \hat{p}_{\rho}}\right)\right)\right)\right\}
$$

in which the left-hand side is some constant depending only on $\gamma, c_{v}$, and $R$ from (2.23). Then (4.10) also holds, and the quadratic part $Q$ is strictly positive definite, that is, there exists $c_{0}>0$ such that

$$
Q \geq c_{0}\left\{\hat{\rho}^{\gamma-2}(\rho-\bar{\rho})^{2}+a^{\prime \prime}(\hat{S}) \hat{\rho}^{\gamma}(S-\bar{S})^{2}\right\} .
$$

Conditions (4.6) and (4.34) can be easily satisfied by choosing

$$
a(S)=a_{0}-\frac{1}{N+1} \ln \left|S_{0}-S\right|, \quad \text { or } \quad \alpha_{0}\left(S^{*}\right)=S_{0}-e^{-(N+1)\left(S^{*}-a_{0}\right)},
$$

for some constants $a_{0}<\underline{S}_{0}^{*}, S_{0}>\bar{S}_{0}$, and sufficiently large $N>0$. Then $a^{\prime}(\bar{S}) \mid S-$ $\bar{S} \mid \leq \frac{1}{N+1}$. Therefore, we have

$$
\begin{aligned}
\tilde{Q} & \leq C\left\{\frac{1}{N+1} \hat{\rho}^{\gamma-2}(\rho-\bar{\rho})^{2}+a^{\prime}(\hat{S}) \hat{\rho}^{\gamma-1}|\rho-\bar{\rho} \| S-\bar{S}|\right\} \\
& \leq \frac{C}{\sqrt{N}}\left\{\hat{\rho}^{\gamma-2}(\rho-\bar{\rho})^{2}+a^{\prime \prime}(\hat{S}) \hat{\rho}^{\gamma}(S-\bar{S})^{2}\right\} \leq \frac{C}{\sqrt{N}} Q
\end{aligned}
$$

which implies that $\tilde{Q}$ is controlled by $Q$ for sufficiently large $N>0$, and hence $\Delta_{j}(W, \bar{W}) \geq 0, j=1,3$.

Also, we find that $h_{j}(x, s), j=1,3$, are uniformly bounded everywhere even at the vacuum states, which means that $h_{j}(x, s), j=1,3$, are integrable in $\Omega_{1}(t) \cup \Omega_{3}(t)$ as $s>0$.

These facts in combination with (4.23) yield

$$
\int_{|x| \leq L} \alpha(W(x, t), \bar{W}(x / t)) d x \leq \int_{|x| \leq L+M(t-\delta)} \alpha(W(x, \delta), \bar{W}(x / \delta)) d x .
$$

Then (4.22) and (4.36) imply (4.13) by taking $\delta \rightarrow 0$. This completes the proof.

From the proof above, especially the proof of Step 4, we can easily deduce the following facts. 
Theorem 4.3. Condition (4.12) on $S^{*}(x, t)$ in Theorem 4.1 can be replaced by

$$
\left|S^{*}(x, t)-\bar{S}^{*}\right| \leq C_{*}, \quad \text { for some } C_{*}>0 .
$$

That is, there exists $C_{*}>0$ such that, for any entropy solution $U(x, t)$ satisfying (4.37), the results of Theorem 4.1 hold.

REMARK 4.1. Theorem 4.3 allows large oscillation of the variables $(\rho, m)$ in the entropy solutions, although we may require moderate oscillation of $S^{*}(x, t)$. If the oscillation of the entropy solutions $U(x, t)$ and $R(x / t)$ is sufficiently small, that is, there exists $C_{*}>0$ such that, when

$$
|W(x, t)-\bar{W}(x / t)| \leq C_{*},
$$

Theorem 4.1 holds even for all adiabatic exponents $\gamma>1$.

REMARK 4.2. Theorem 4.1 can be easily extended to system (2.20) with general constitutive relations $p=p\left(\rho, S^{*}\right), \theta=\theta\left(\rho, S^{*}\right)$, and $e=e\left(\rho, S^{*}\right)$ satisfy $(2.21)-(2.22)$ and certain asymptotic properties near the vacuum, by carefully estimating $Q$ and $\tilde{Q}$ in Step 4 above.

For more general Riemann solutions containing a contact discontinuity, one can follow the approach in Chen-Frid [6] to prove the stability of the Riemann solutions in the class of entropy solutions in $B V$ away from the vacuum.

As a corollary, we conclude

THEOREM 4.4. Let $R(x / t)$ be the Riemann solution of (2.20), (2.23), and (2.3), consisting of one or two rarefaction waves, constant states, and possible vacuum states, as constructed in §2.2. Let $U(x, t)$ be any entropy solution of (2.20), (2.23), (2.2), and (2.4), defined by Definition 4.1, satisfying either (4.12) or (4.37). Then $U(x, t)$ is asymptotically stable under the initial perturbation $P_{0}(x) \in L^{1} \cap L^{\infty}$ in the sense of

$$
\lim _{t \rightarrow \infty} \int_{|\xi| \leq L} \alpha((\rho, m, H)(\xi t, t),(\bar{\rho}, \bar{m}, \bar{H})(\xi)) d \xi=0, \quad \text { for any } L>0 .
$$

5. Vacuum States and the Compressible Navier-Stokes Equations. Finally, we discuss the vacuum states for the compressible Navier-Stokes equations and present some techniques to obtain a lower bound of the density for a free boundary problem of the compressible Navier-Stokes equations that connects the viscous compressible non-isentropic fluids to a surrounding vacuum state with spherical symmetry and initial data of large oscillation. The lower bound of the density is essential for the compressible Navier-Stokes equations as pointed out in Hoff-Serre [20]. These techniques have been applied to establishing the existence of global solutions and the finiteness of propagation speed of the free boundary for the compressible nonisentropic Navier-Stokes equations in Chen-Krakta [9].

For the one-dimensional compressible Navier-Stokes equations for isentropic fluids having density-dependent viscosity with a free boundary connected to a vacuum state, and related problems, see recent papers Liu-Xin-Yang [30] and Jiang-Xin-Zhang [22], and the earlier references cited therein. Also see Okada [39], Okada-Makino [40], and Lions [27] for related references for the isentropic fluids. 
For the Cauchy problem or the initial-boundary value problem for one-dimensional compressible non-isentropic Navier-Stokes equations with constant viscosity, if $\rho_{0}(x) \geq$ $c_{0}>0$, then $\rho(x, t) \geq c(t)>0$; see Kazhikov [23] and Kazhikov-Shelukhin [24] for nondiscontinuous initial data and Chen-Hoff-Trivisa [10] and the references cited therein for discontinuous initial data of large oscillation. Also see Jiang [21] for spherically symmetric solutions to the compressible non-isentropic Navier-Stokes equations with non-discontinuous initial data of large oscillation outside the solid core. For multidimensional Navier-Stokes equations with small initial data, see Matsumura-Nishida $[32,33]$ for smooth initial data and Hoff [19] for discontinuous initial data.

We are concerned with the compressible non-isentropic fluids between two spherical boundaries: the fixed boundary is the solid core surface with static zero velocity and thermally insulation; and the free boundary connects the compressible nonisentropic fluids to a surrounding vacuum state with free stress and zero heat flux. The fluids are initially assumed to fill with a finite volume, zero density at the free boundary, and bounded positive density and temperature between the solid core and the initial position of the free boundary. For physical significance and mathematical interests for such free boundaries, we refer to Nishida [37, 38], Antontsev-KazhikhovMonakhov [1], and the references cited therein.

The spherical symmetric solutions $(\rho, v, e)(r, t), r=\sqrt{x_{1}^{2}+\cdots+x_{n}^{2}}$, for the compressible Navier-Stokes equations are governed by

$$
\left\{\begin{array}{l}
\partial_{t} \rho+\partial_{r}(\rho v)+\frac{n-1}{r} \rho v=0 \\
\rho\left(\partial_{t} v+v \partial_{r} v\right)+\partial_{r} p=(\lambda+2 \mu)\left(\partial_{r r} v+\frac{n-1}{r} \partial_{r} v-\frac{n-1}{r^{2}} v\right) \\
\rho\left(\partial_{t} e+v \partial_{r} e\right)+p\left(\partial_{r} v+\frac{n-1}{r} v\right) \\
\quad=\kappa\left(\partial_{r r} e+\frac{n-1}{r} \partial_{r} e\right)+\lambda\left(\partial_{r} v+\frac{n-1}{r} v\right)^{2}+2 \mu\left(\left(\partial_{r} v\right)^{2}+\frac{n-1}{r^{2}} v^{2}\right)
\end{array}\right.
$$

where we have denoted

$$
\rho(\vec{x}, t)=\rho(r, t), \quad \vec{v}(\vec{x}, t)=v(r, t) \frac{\vec{x}}{r}, \quad e(\vec{x}, t)=e(r, t) .
$$

Here $\rho, e$, and $\vec{v}=\left(v_{1}, \cdots, v_{n}\right)$ are the density, the internal energy, and the velocity, respectively; $\lambda$ and $\mu$ are the constant viscosity coefficients, $\mu>0, \lambda+2 \mu / n \geq 0 ; \kappa>0$ is the constant related to the heat conductivity coefficient. We focus on polytropic ideal fluids in which the internal energy, the temperature, and the pressure have relations $(2.23)$.

To handle this problem, it is convenient to reduce the problem in the global Eulerian space-time coordinates $(r, t)$ to the problem in the local Lagrangian masstime coordinates $(x, t)$ moving with the fluid, via the transformation:

$$
x=\int_{1}^{r(x, t)} s^{n-1} \rho(s, t) d s, \quad \text { or } \quad r(x, t)=r(x, 0)+\int_{0}^{t} v(r(x, \tau), \tau) d \tau .
$$

It is easy to check that $x=\int_{1}^{r(x, 0)} s^{n-1} \rho_{0}(s) d s$. Without loss of generality, we assume $\int_{1}^{a} s^{n-1} \rho_{0}(s) d x=1$, so that the transformation is from the region $\{(r, t) \mid 1 \leq r \leq$ $r(t), 0 \leq t \leq T\}$ under consideration into the region $\{(x, t) \mid 0 \leq x \leq 1,0 \leq t \leq T\}$.

With this change of coordinates, we can rewrite system (5.1) into the following system:

$$
\left\{\begin{array}{l}
\partial_{t} \rho=-\rho^{2} \partial_{x}\left(r^{n-1} v\right) \\
\partial_{t} v=r^{n-1} \partial_{x} \sigma \\
\partial_{t} e=\kappa \partial_{x}\left(\rho r^{2 n-2} \partial_{x} e\right)+\sigma \partial_{x}\left(r^{n-1} v\right)-2 \mu(n-1) \partial_{x}\left(r^{n-2} v^{2}\right)
\end{array}\right.
$$


where $\sigma=(\lambda+2 \mu) \rho \partial_{x}\left(r^{n-1} v\right)-p$.

The initial conditions can be written in the form

$$
\left.(\rho, v, e)\right|_{t=0}=\left(\rho_{0}, v_{0}, e_{0}\right)(x), \quad 0 \leq x \leq 1 .
$$

The free and fixed boundary conditions then are

$$
v(0, t)=0, \quad\left(\rho \partial_{x} e\right)(0, t)=\left(\rho \partial_{x} e\right)(1, t)=0, \quad \sigma(1, t)=0 .
$$

To articulate the assumption that there is no initial cavity with gas residing in the bounded region, we assume that there exist a decreasing nonnegative function $\Lambda(x)$ with $\Lambda(0)=0, \int_{0}^{1} \Lambda^{-1}(x) d x<\infty$, and a constant $C_{0}>0$ such that

$$
C_{0}^{-1} \Lambda(x) \leq \rho_{0}(x) \leq C_{0} \Lambda(x), \quad x \in[0,1] .
$$

To present the techniques more clearly, we show the following proposition for illustration.

Proposition 5.1. Any $C^{2}$ solution of problem (5.3)-(5.6) between the solid core and the free boundary does not develop the vacuum as time evolves.

Proof. We divide the description of the techniques into three steps. It suffices to prove that the vacuum states are not developed in any finite time interval $[0, T]$.

Step 1. Claim: there exists $C>0$ depending only on the initial data and $T>0$ such that

$$
0 \leq \int_{0}^{1} E(x, t) d x+\int_{0}^{t} \int_{0}^{1} \rho\left(r^{n-1} \partial_{x} e / e\right)^{2} d x d s \leq C, \quad t \in[0, T],
$$

where $E(x, t)=\frac{1}{2} v^{2}(x, t)+R \Phi(1 / \rho(x, t))+\Phi(e(x, t)) \geq 0$, with $\Phi(u)=u-1-\log (u) \geq$ 0 .

For system (5.3), after applying simple algebraic operations of multiplication and addition, integrating over $[0,1] \times[0, t]$, and applying the boundary conditions $(5.5)$, we have

$$
\begin{aligned}
0 & \leq \int_{0}^{1} E(x, t) d x+\kappa \int_{0}^{t} \int_{0}^{1} \rho\left(r^{n-1} \partial_{x} e / e\right)^{2} d x d s \\
& \leq \int_{0}^{1} E(x, 0) d x+\int_{0}^{t}\left(R\left(r^{n-1} v\right)(1, s)-2 \mu(n-1)\left(r^{n-2} v^{2}\right)(1, s)\right) d s .
\end{aligned}
$$

To arrive at (5.7), the main point is to estimate the last integral of (5.8). For this purpose, we introduce $p(t)=\sqrt{r^{n}(1, t) / n}$, and apply the identities

$$
2 p(t) p^{\prime}(t)=\left(r^{n-1} v\right)(1, t), \quad \frac{4}{n}\left(p^{\prime}\right)^{2}(t)=\left(r^{n-2} v^{2}\right)(1, t),
$$

and the inequality $2 p(t) p^{\prime}(t) \leq \alpha\left(p^{\prime}\right)^{2}(t)+\frac{1}{\alpha} p^{2}(t)$ with $\alpha=4 \mu(n-1) /(n R)$. Then, from (5.8), we have

$$
-C \equiv-\frac{\alpha}{R} \int_{0}^{1} E(x, 0) d x \leq \int_{0}^{t} p^{2}(s) d s-\alpha^{2} \int_{0}^{t}\left(p^{\prime}\right)^{2}(s) d s,
$$

which implies

$$
\int_{0}^{t}\left(p^{\prime}\right)^{2}(s) d s \leq \frac{C+2 t p^{2}(0)}{\alpha^{2}}+\int_{0}^{t} \frac{2 s}{\alpha^{2}} \int_{0}^{s}\left(p^{\prime}\right)^{2}(\tau) d \tau d s
$$


where we have used the inequality $p^{2}(t)=\left(p(0)+\int_{0}^{t} p^{\prime}(s) d s\right)^{2} \leq 2 p^{2}(0)+2 t \int_{0}^{t}\left(p^{\prime}\right)^{2}(s) d s$.

Gronwall's inequality for (5.9) yields $p^{2}(t)+\int_{0}^{t}\left(p^{\prime}\right)^{2}(s) d s \leq C$, which implies

$$
r(x, t) \leq r(1, t) \leq C, \quad \int_{0}^{t} v^{2}(1, s) d s \leq C .
$$

This implies the finiteness of propagation speed of the free boundary and yields (5.7) from (5.8).

As a corollary of (5.7) and the convexity of $\Phi(u)$, there exists $C>0$ such that

$$
C^{-1} \leq \frac{1}{\alpha} \int_{0}^{\alpha} \rho^{-1}(x, t) d x \leq C, \quad C^{-1} \leq \frac{1}{\alpha} \int_{0}^{\alpha} e(x, t) d x \leq C .
$$

Hence there exist $a(t) \in(0,1)$ and $b_{x}(t) \in(0, \max (x, 1 / 2))$ such that

$$
\begin{aligned}
& C^{-1} \leq \rho^{-1}(a(t), t)=\int_{0}^{1} \rho^{-1}(x, t) d x \leq C, \\
& C^{-1} \leq e^{-1}\left(b_{x}(t), t\right)=\int_{0}^{\max (x, 1 / 2)} e(y, t) d y \leq C .
\end{aligned}
$$

Step 2. Claim: There exists $C>0$ such that

$$
\rho(x, t) \leq C \rho(x, 0) \text {. }
$$

The momentum equation can be rewritten into

$$
\frac{1}{\lambda+2 \mu} r^{1-n} \partial_{t} v=-\partial_{x t}(\log \rho)-\frac{R}{\lambda+2 \mu} \partial_{x}(\rho e) .
$$

Integrating the identity over the region $[a(t), x] \times[0, t]$ if $a(t) \leq x$, or $[x, a(t)] \times[0, t]$ if $x \leq a(t)$, and then taking exponentials of both sides, we obtain

$$
\frac{\rho(x, 0)}{\rho(x, t)} Y(t) B(x, t) \frac{\rho(a(t), t)}{\rho(a(t), 0)}=\exp \left\{\frac{R}{\lambda+2 \mu} \int_{0}^{t}(\rho e)(x, s) d s\right\}
$$

where

$$
\begin{aligned}
Y(t) & =\exp \left\{\frac{R}{\lambda+2 \mu} \int_{0}^{t}(\rho e)(a(t), s) d s\right\} \\
B(x, t) & =\exp \left\{-\frac{1}{\lambda+2 \mu} \int_{a(t)}^{x} \int_{0}^{t}\left(r^{1-n} \partial_{t} v\right)(y, s) d s d y\right\} .
\end{aligned}
$$

Furthermore, multiplying (5.14) by $\frac{R}{\lambda+2 \mu}(\rho e)(x, t)$, integrating over $[0, t]$, and factoring out the full derivative of the right-hand side, we obtain the following identity

$$
\frac{R}{\lambda+2 \mu} \int_{0}^{t} \rho(x, 0) e(x, s) Y(s) B(x, s) \frac{\rho(a(s), s)}{\rho(a(s), 0)} d s=\frac{\rho(x, 0)}{\rho(x, t)} Y(t) B(x, t) \frac{\rho(a(t), t)}{\rho(a(t), 0)}-1 .
$$

It is easy to check

$$
C^{-1} \leq B(x, t) \leq C, \quad Y(t) \geq 1
$$


Set $\tilde{Y}(t)=Y(t) / \rho(a(t), 0)$. Then identity (5.15) implies

$$
\tilde{Y}(t) / \rho(x, t) \leq C / \rho(x, 0)+C \int_{0}^{t} e(x, s) \tilde{Y}(s) d s
$$

which, by integrating it in $x \in[0,1]$ and using (5.11), yields

$$
\tilde{Y}(t) \leq C+C \int_{0}^{t} \tilde{Y}(s) d s
$$

Gronwall's inequality yields

$$
Y(t) \leq \tilde{Y}(t) \rho(a(t), t) \leq C \rho(a(t), 0) \leq C .
$$

Then identity (5.14) implies

$$
\begin{aligned}
\rho(x, t) & \leq \rho(x, t) \exp \left\{\frac{R}{\lambda+2 \mu} \int_{0}^{t} \rho(x, s) e(x, s) d s\right\} \\
& =\rho(x, 0) Y(t) B(x, t) \frac{\rho(a(t), t)}{\rho(a(t), 0)} \leq C \rho(x, 0) .
\end{aligned}
$$

Step 3. Claim: There exists $C>0$ such that $\rho(x, t) \geq C^{-1} \rho(x, 0)$.

Since

$$
\begin{aligned}
e(x, t) & \leq\left(\sqrt{e\left(b_{x}(t), t\right)}+\int_{b_{x}(t)}^{x} \frac{\partial_{x} e(y, t)}{2 \sqrt{e(y, t)}} d y\right)^{2} \\
& \leq C+C \int_{0}^{1} \frac{\left(\rho\left(\partial_{x} e\right)^{2}\right)(x, t)}{e^{2}(x, t)} d x\left|\int_{b_{x}(t)}^{x} \frac{e(y, t)}{\rho(y, t)} d y\right|,
\end{aligned}
$$

we conclude from (5.13)-(5.15) that

$$
\begin{aligned}
\frac{\rho(x, 0)}{\rho(x, t)} & \leq C \frac{\rho(x, 0)}{\rho(x, t)} \rho(a(t), t) \tilde{Y}(t) B(x, t) \\
& =C\left(1+\frac{R}{\lambda+2 \mu} \int_{0}^{t} \rho(x, 0) \rho(a(s), s) e(x, s) \tilde{Y}(t) B(x, s) d s\right) \\
& \leq C+C \rho(x, 0) \int_{0}^{t} \int_{0}^{1} \frac{\left(\rho\left(\partial_{x} e\right)^{2}\right)(y, s)}{e^{2}(y, s)} d y\left|\int_{b_{x}(s)}^{x} \frac{e(y, s)}{\rho(y, s)} d y\right| d s .
\end{aligned}
$$

We have two cases:

Case 1. $x \leq 1 / 2: \rho(1 / 2,0) \leq \Lambda(1 / 2) \leq C \Lambda(x) \leq C \rho(x, 0)$. Then inequality (5.18) implies

$$
\max _{x \in[0,1 / 2]} \rho(x, t)^{-1} \leq C+C \int_{0}^{t} \int_{0}^{1} \frac{\left(\rho\left(\partial_{x} e\right)^{2}\right)(y, s)}{e^{2}(y, s)} \max _{y \in[0,1 / 2]} \rho(y, t)^{-1} d s,
$$

which implies $\rho^{-1}(x, t) \leq C$, for $x \in[0,1 / 2]$, by Gronwall's inequality.

Case 2. $1 / 2<x<1: \rho(1 / 2,0) \leq \Lambda(1 / 2) \leq C \Lambda(x) \leq C \rho(x, 0)$. Using (5.11), the result of Case 1 , and inequality (5.18) yields

$$
\frac{\rho(x, 0)}{\rho(x, t)} \leq C+C \int_{0}^{t} \int_{0}^{1} \frac{\left(\rho\left(\partial_{x} e\right)^{2}\right)(y, s)}{e^{2}(y, s)} \max _{y \in[1 / 2,1)} \frac{\rho(y, 0)}{\rho(y, t)} d y d s .
$$


Gronwall's inequality implies

$$
\max _{x \in[1 / 2,1)} \frac{\rho(x, 0)}{\rho(x, t)} \leq C,
$$

which yields the claim with the aid of (5.19). This completes the proof.

In fact, the solutions of this problem generally develop a singularity near the free boundary, so that one can not expect a global $C^{2}$ solution in general, and the proposition above is just for illustration to make the points more clearly. To avoid this difficulty, in Chen-Krakta [9], we use the semi-discrete difference scheme to construct approximate solutions, and then we apply the techniques we explained above to obtain the uniform lower bound for the approximate density, which is essential to obtain other essential estimates to conclude the compactness of the approximate solutions and the lower bound of the density in the solutions. For more details, see [9].

Acknowledgments. The author would like to thank Hermano Frid and Milan Krakta for helpful discussions. Gui-Qiang Chen's research was supported in part by the National Science Foundation under Grants DMS-9971793, INT-9987378, and INT-9726215.

\section{REFERENCES}

[1] S. N. Antontsev, A. V. Kazhikhov, and V. N. Monakhov, Boundary Value Problems in Mechanics of Nonhomogeneous Fluids, North-Holland, Amsterdam-New York, 1990.

[2] G. Anzellotti, Pairings between measures and bounded functions and compensated compactness, Ann. Mat. Pura Appl., 135:4 (1983), pp. 293-318.

[3] T. Chang and L. Hsiao, The Riemann Problem and Interaction of Waves in Gas Dynamics, Longman Scientific \& Technical, Harlow; and John Wiley \& Sons, Inc., New York, 1989.

[4] G.-Q. CHEN, Convergence of the Lax-Friedrichs scheme for isentropic gas dynamics (III), Acta Math. Sci., 6 (1986), pp. 75-120 (in English); and 8 (1988), pp. 243-276 (in Chinese).

[5] G.-Q. Chen AND H. FRID, Divergence-measure fields and hyperbolic conservation laws, Arch. Rational Mech. Anal., 147 (1999), pp. 89-118.

[6] G.-Q. CHEN AND H. FRID, Uniqueness and asymptotic stability of Riemann solutions for the compressible Euler equations, Trans. Amer. Math. Soc., to appear (2000).

[7] G.-Q. CHEN AND H. FRID, Large-time behavior of entropy solutions of conservation laws, J. Diff. Eqs., 152 (1999), pp. 308-357.

[8] G.-Q. Chen AND P. KAN, in preparation.

[9] G.-Q. ChEN AND M. KRAKTA, Global solutions to the Navier-Stokes equations for nonisentropic fluids with large spherically symmetric initial data and a free boundary connected to a vacuum, preprint, December 1999.

[10] G.-Q. Chen, D. HofF, AND K. TRIVISA, Global solutions of the compressible Navier-Stokes equations with large discontinuous initial data, Commun. Partial Diff. Eqs., to appear (2000).

[11] G.-Q. Chen And P. LeFloch, Compressible Euler equation with a general pressure law, Arch. Rational Mech. Anal., 153 (2000), pp. 221-259.

[12] G.-Q. CHEN AND M. RASCLE, Initial layers and uniqueness of weak entropy solutions to hyperbolic conservation laws, Arch. Rational Mech. Anal., 153 (2000), pp. 205-220.

[13] R. Courant And K. O. Friedrichs, Supersonic Flow and Shock Waves, Interscience, New York, 1948.

[14] C. M. DAFERmos, Entropy and the stability of classical solutions of hyperbolic systems of conservation laws, in Recent Mathematical Methods in Nonlinear Wave Propagation (Montecatini Terme, 1994), Lecture Notes in Math. 1640, Springer, Berlin, 1996, pp. 48-69.

[15] C. M. Dafermos, Hyperbolic Systems of Conservation Laws from Continuum Physics, Springer-Verlag, New York, 1999.

[16] X. Ding, G.-Q. Chen, AND P. Luo, Convergence of the Lax-Friedrichs scheme for the isentropic gas dynamics (I)-(II), Acta Math. Sci., 5 (1985), pp. 483-500 \& 501-540 (in English); 7 (1987), pp. $467-480 \& 8$ (1989), pp. 61-94 (in Chinese). 
[17] R. DiPERNA, Uniqueness of solutions to hyperbolic conservation laws, Indiana U. Math. J., 28 (1979), pp. 137-188.

[18] R. DiPERnA, Convergence of the viscosity method for isentropic gas dynamics, Commun. Math. Phys., 91 (1983), pp. 1-30.

[19] D. HoFF, Discontinuous solutions of the Navier-Stokes equations for multidimensional heatconducting fluids, Arch. Rational Mech. Anal., 139 (1997), pp. 303-354.

[20] D. HoFF AND D. SERre, The failure of continuous dependence on initial data for the NavierStokes equations of compressible flow, SIAM J. Appl. Math., 51 (1991), pp. 887-898.

[21] S. JIANG, Global spherically symmetric solutions to the equations of a viscous polytropic ideal gas in an exterior domain, Comm. Math. Phys., 178 (1996), pp. 339-374.

[22] S. JIANG, Z. XIN, AND P. ZHANG, Global weak solutions to 1 D compressible isentropic NavierStokes equations with density-dependent viscosity, preprint, 1999.

[23] A. V. KazhikHov, On the theory of initial-boundary-value problems for the equations of onedimensional nonstationary motion of a viscous heat-conductive gas, Din. Sploshnoi Sredy, 50 (1981), pp. 37-62 (Russian).

[24] A. V. Kazhikhov ANd V. V. Shelukhin, Unique global solution with respect to time of initialboundary-value problems for one-dimensional equations of a viscous gas, J. Appl. Math. Mech., 41 (1977), pp. 273-282.

[25] P. D. LAx, Hyperbolic Systems of Conservation Laws and the Mathematical Theory of Shock Waves, CBMS 11, SIAM, Philadelphia, 1973.

[26] L. W. LIN, On the vacuum state for the equations of isentropic gas dynamics, J. Math. Anal. Appl., 121 (1987), pp. 406-425.

[27] P.-L. Lions, Mathematical Topics in Fluid Mechanics, Vols. 1-2, Oxford University Press, New York, 1996 \& 1998.

[28] P. L. Lions, B. Perthame, and E. Tadmor, Kinetic formulation for the isentropic gas dynamics and p-system, Commun. Math. Phys., 163 (1994), pp. 415-431.

[29] P. L. Lions, B. Perthame, ANd P. E. Souganidis, Existence and stability of entropy solutions for the hyperbolic systems of isentropic gas dynamics in Eulerian and Lagrangian coordinates, Comm. Pure Appl. Math., 49 (1996), pp. 599-638.

[30] T.-P. LIU, Z. XIN, AND T. YANG, Vacuum states for compressible flow, Contin. Dynamic Systems, 4 (1988), pp. 1-32.

[31] T.-P. LIU AND J. A. SMOLleR, On the vacuum state for the isentropic gas dynamics equations, Adv. Appl. Math., 1 (1980), pp. 345-359.

[32] A. Matsumura AND T. Nishida, The initial-value problem for the equations of motion of viscous and heat-conductive gas, J. Math. Kyoto Univ., 20 (1980), pp. 67-104.

[33] A. MATSUmURA AND T. Nishida, Initial boundary value problems for the equations of motion of compressible viscous and heat-conductive fluids, Comm. Math. Phys., 89 (1983), pp. 445-464.

[34] C. S. Morawetz, On a weak solution for a transonic flow problem, Comm. Pure Appl. Math., 38 (1985), pp. 797-818.

[35] C. S. MORAWETZ, On steady transonic flow by compensated compactness, Meth. Appl. Anal., 2 (1995), pp. 257-268.

[36] C. S. MoRAWETZ, On the non-existence of continuous transonic flows past profiles I,II,III, Comm. Pure Appl. Math., 9 (1956), pp. 45-68; 10 (1957), pp. 107-131; 11 (1958), pp. 129-144.

[37] T. NishidA, Equations of fluid dynamics-free surface problems, Comm. Pure Appl. Math., 39 (1986), pp. 221-238.

[38] T. NisHidA, Equations of motion of compressible viscous fluids, in Patterns and WavesQualitative Analysis of Nonlinear Differential Equations, T. Nishida, M. Mimura, and H. Fujii, eds., North-Holland, Amsterdam-New York, 1986, pp. 97-128.

[39] M. OKADA, Free boundary value problems for the equation of one-dimensional motion of viscous gas, Japan J. Appl. Math., 6 (1989), pp. 161-177.

[40] M. OKADA AND T. MAKINO, Free boundary problem for the equation of spherically symmetrical motion of viscous gas, Japan J. Appl. Math., 10 (1993), pp. 219-235.

[41] L. Schwartz, Théorie des Distributions, Actualites Scientifiques et Industrielles 1091 \& 1122 , Herman, Paris, 1950 \& 1951.

[42] J. Smoller, Shock Waves and Reaction-Diffusion Equations, 2nd Edition, Springer-Verlag, New York, 1994.

[43] Z. XIN, Blow-up of smooth solutions to the compressible Navier-Stokes equations with compact density, Comm. Pure Appl. Math., 51 (1998), pp. 229-240.

[44] T. YANG, Z.-A. YAO, AND C. ZHU, Compressible Navier-Stokes equations with densitydependent viscosity and vacuum, preprint, 1999. 
